\title{
Multiple Criteria Ranking and Choice with All Compatible Minimal Cover Sets of Decision Rules
}

\author{
Miłosz Kadziński ${ }^{a, *}$, Roman Słowiński ${ }^{\mathrm{a}, \mathrm{b}}$, Salvatore Greco ${ }^{\mathrm{c}, \mathrm{d}}$ \\ ${ }^{a}$ Institute of Computing Science, Poznań University of Technology, Poznań, Poland \\ ${ }^{b}$ Systems Research Institute, Polish Academy of Sciences, Warsaw, Poland \\ ${ }^{c}$ Department of Economics and Business, University of Catania, Catania, Italy \\ ${ }^{d}$ Portsmouth Businees School, Centre of Operations Research and Logistics (CORL), University of Portsmouth, United Kingdom
}

\begin{abstract}
We introduce a new multiple criteria ranking/choice method that applies Dominance-based Rough Set Approach (DRSA) and represents the Decision Maker's (DM's) preferences with decision rules. The DM provides a set of pairwise comparisons indicating whether an outranking (weak preference) relation should hold for some pairs of reference alternatives. This preference information is structured using the lower and upper approximations of outranking $(S)$ and non-outranking $\left(S^{c}\right)$ relations. Then, all minimal-cover (MC) sets of decision rules being compatible with this preference information are induced. Each of these sets is supported by some positive examples (pairs of reference alternatives from the lower approximation of a preference relation) and it does not cover any negative example (pair of alternatives from the upper approximation of an opposite preference relation). The recommendations obtained by all $\mathrm{MC}$ sets of rules are analyzed to describe pairwise outranking and non-outranking relations, using probabilistic indices (estimates of probabilities that one alternative outranks or does not outrank the other). Furthermore, given the preference relations obtained in result of application of each $\mathrm{MC}$ set of rules on a considered set of alternatives, we exploit them using some scoring procedures. From this, we derive the distribution of ranks attained by the alternatives. We also extend the basic approach in several ways. The practical usefulness of the method is demonstrated on a problem of ranking Polish cities according to their innovativeness.
\end{abstract}

Keywords: Decision analysis, Decision rule, Multiple criteria ranking, Pairwise comparisons, Dominance-based Rough Set Approach, Scoring procedures

\section{Introduction}

In this paper, we consider choice and ranking problems where alternatives are evaluated on multiple, often conflicting, criteria. Choice problems are oriented toward selecting a subset of the most prevailing

\footnotetext{
*Corresponding author: Institute of Computing Science, Poznań University of Technology, Piotrowo 2, 60-965 Poznań, Poland. Tel.: +48-61 665 3022, Fax: +48-61 8771525

Email address: milosz.kadzinski@cs.put.poznan.pl (Miłosz Kadziński)
} 
alternatives, whereas in ranking problems one aims at imposing a preference order on the set of alternatives. The only conclusion which can be derived from the analysis of performances of the considered alternatives on multiple criteria is the dominance relation among them. Leaving many alternatives incomparable, this relation prevents, however, their clear ranking or straightforward discrimination between alternatives that should be selected and neglected. Thus, to work out a recommendation, the Decision Maker (DM) needs to enrich the dominance relation by providing some extra preference information which is subsequently transformed into a mathematical preference model. The application of the preference model induces a preference structure on the set of alternatives. The ranking or choice recommendation can be derived from its proper exploitation. In Multiple Criteria Decision Aiding (MCDA), there exist three basic ways of modeling preference information coming from the DM: value functions (Keeney and Raiffa, 1976), relational systems (Roy, 1990), or "if ..., then ..." decision rules (Greco et al., 2001a, 2005; Słowiński et al., 2012).

These models need to faithfully represent the elements of DM's value system. In this perspective, they can be tuned using either direct or indirect preference statements. Since the previous need a considerable cognitive effort on the DM's part, the recent decision aiding methods are designed so that to accommodate indirect or incomplete preference information. In the context of multiple criteria ranking and choice, such preference information is composed of some exemplary decisions concerning a small subset of reference alternatives. Although these judgments may have different forms (for a review, see (Corrente et al., 2013)), the majority of methods employ pairwise comparisons.

\subsection{Robustness analysis in value- and outranking-based multiple criteria ranking and choice methods}

With indirect and incomplete specification of preferences, there are typically several instances of the preference model (i.e., functions, relations, or sets of rules) that are consistent with the preference statements. While all such compatible instances reproduce the preference information provided by the DM for reference alternatives, the recommendation delivered for the non-reference ones may vary significantly from one instance to another. The potential diversity of the suggested recommendation motivated the development of a framework for robustness analysis. Dealing with the plurality of compatible preference model instances in the context of ranking and choice problems has been already considered for two out of three preference models used in MCDA: a value function and an outranking relation.

When analyzing the robustness of recommendation obtained with a value-based preference model, we may identify such pairs of alternatives that the comprehensive value of the first one is at least as good as that of the second one for all value functions, and strictly higher for some value functions (Hazen, 1986; White et al., 1984). A similar result in the setting of Robust Ordinal Regression (ROR) corresponds to the necessary and 
possible preference relations (Corrente et al., 2012; Greco et al., 2008). Moreover, we may take into account ranks attained by the alternatives by indicating either potentially optimal alternatives (i.e., these which can by ranked first by some compatible value function) (Hazen, 1986; Lee et al., 2002) or, more generally, the whole range of ranks for each alternative by conducting extreme ranking analysis (Kadziński et al., 2012). Finally, within the framework of Stochastic Ordinal Regression (SOR), one may estimate probabilities of both preference relations and attaining some rank, using the Monte Carlo simulation (Kadziński and Tervonen, 2013; Lahdelma and Salminen, 2012).

When it comes to outranking-based ranking and choice methods, the robustness concern has been raised by (Dias and Clímaco, 2002), (Greco et al., 2011), and (Kadziński et al., 2012). These approaches admit indirect and partial preference information concerning parameters of the model used in ELECTRE (see, e.g., (Figueira et al., 2013)) or PROMETHEE (see, e.g., (Brans and Mareschal, 1994)). Having constructed a set of relational systems compatible with the DM's preferences, they verify the possibility and necessity of an outranking relation for each pair of alternatives by checking if it holds for, respectively, at least one or all admissible combinations of parameter values.

\subsection{Rule induction algorithms for multiple criteria ranking and choice}

The above review proves that robustness analysis has been widely used as a decision aiding tool within value- and outranking-based ranking and choice methods. Nevertheless, it has not received due attention in the context of decision rules. This model has been introduced to decision analysis several years ago, quickly gaining popularity because of its explanation potential and recommendation formulated in a natural language. The use of decision rules in MCDA is inseparably connected with Dominance-based Rough Set Approach (DRSA) (Greco et al., 2001a; Słowiński et al., 2012) (for some recent advances or applications of DRSA, see, e.g., (An and Tong, 2010; Augeri et al., 2015; Chakhar and Saad, 2012; Huang et al., 2012; Li et al., 2013, 2015a,b)). It structures the data so that sets of alternatives (in case of sorting problems) or sets of pairs of alternatives (in case of ranking and choice problems) are represented by the lower and upper approximations of decision classes or preference relations, respectively (Pawlak and Słowiński, 1994).

In adaptation of DRSA to multiple criteria ranking (Greco et al., 1999a), the DM provides a set of pairwise comparisons indicating whether an outranking (weak preference) relation should hold $(S)$ or not $\left(S^{c}\right)$ for some reference alternatives. Decision rules which are induced from the approximations of comprehensive outranking $(S)$ and non-outranking $\left(S^{c}\right)$ relations concern pairs of alternatives. Their application on the set of alternatives yields a specific preference relation in this set. This relation needs to be further exploited with some ranking method that arranges the alternatives in a preference order (see, e.g., (Bouyssou and Perny, 1992; Dias and 
Lamboray, 2010; Szelag et al., 2013, 2014)).

Many algorithms for induction of decision rules have been introduced in the context of multiple criteria ranking approached with DRSA. The vast majority of these algorithms generates a minimal-cover (MC) set of minimal decision rules (Błaszczyński et al., 2011; Greco et al., 1999a; Szelag et al., 2013, 2014). In this way, pairs of alternatives from the lower or upper approximations of outranking and non-outranking relations are described with the set of most general, complete and non-redundant "if ..., then ..." statements. However, there are usually multiple sets of rules satisfying these properties, and the existing algorithms select a single one in an arbitrary pre-defined way. Obviously, the ranking or choice recommendation that can be obtained for any compatible set of rules can vary significantly.

\subsection{Content and plan of the paper}

The aim of this paper is to introduce an approach for multiple criteria ranking and choice with all MC sets of rules compatible with the DM's indirect and incomplete preference information. Analogously to (An and Tong, 2009; Greco et al., 1999a, 2011; Szelag et al., 2013, 2014), we expect the DM to provide a set of pairwise comparisons stating the truth $(S)$ or falsity $\left(S^{c}\right)$ of the outranking relation for some reference alternatives. Thus exhibited preference information is treated as deterministic, and structured using the lower and upper approximations of outranking and non-outranking relations. Then, all MC sets of decision rules being compatible with this preference information are induced from the structured information, such that rules suggesting $S$ are induced with the hypothesis that the lower approximation of $S$ provides positive examples and the upper approximation of $S^{c}$ provides negative examples, and vice versa in case of inducing rules suggesting $S^{c}$. The compatibility of inferred rule sets with the exhibited DM's preference information is due to the fact that these sets cover all pairs of reference alternatives from the lower approximation of $S$ and $S^{c}$, respectively. In this regard, analogously to other MCDA methods based on indirect preference information, the supplied pairwise comparisons constrain the flexibility of compatible preference model instances. In our case, a compatible instance of the preference model is a minimal set of minimal rules covering all pairs of reference alternatives compared by the DM and included in lower approximations of $S$ and $S^{c}$ - it is called MC set of rules.

The recommendations obtained by all MC sets of rules are analyzed to describe the stability of outranking and non-outranking by means of the necessary and the possible, as well as by the probabilistic indices (estimates of probabilities that one alternative outranks or does not outrank the other). To pass from the robustness analysis of the preference relations imposed on the set of alternatives to the recommended ranking/choice, we exploit them using different scoring procedures and derive the distribution of ranks attained 
by the alternatives. We also extend the basic approach in several ways.

The approach presented in this paper can be seen as a rule-based counterpart of Robust and Stochastic Ordinal Regression methods with value- (Greco et al., 2008; Kadziński and Tervonen, 2013) and outrankingbased (Greco et al., 2011) preference models. In this regard, our main contribution is in the phase of construction of the DM's preference model. Precisely, we extend the existing rule-based methods for multiple criteria ranking and choice (Błaszczyński et al., 2011; Greco et al., 1999a; Fortemps et al., 2008; Szelag et al., 2013, 2014) to take into account all compatible MC set of rules, and not only one such set. Note, however, that when using value- or outranking-based preference model, either all compatible preference model instances are considered implicitly by taking into account a set of Linear Programming (LP) constraints or one analyzes a proper subset of all instances obtained with the Monte Carlo simulation. On the contrary, when dealing with decision rules, all compatible MC sets of rules need to be constructed explicitly.

Furthermore, our proposal is a ranking/choice counterpart of the DRSA-based framework for robustness analysis for multiple criteria sorting (ordinal classification) problems (Kadziński et al., 2014b). In this regard, the main innovation of the paper is in the phase of exploitation of the preference model. It consists in adapting different concepts and measures of robustness to the specific context of DRSA applied to ranking/choice problems. Thus, instead of investigating the stability of class assignments as in (Kadziński et al., 2014b), we rather focus on the pairwise preference relations and ranks attained by the alternatives.

Finally, analogously to the approach presented by (Greco et al., 2013), we are putting DRSA and ROR together for multiple criteria ranking and choice. However, instead of providing the explanations of the necessary and possible preference relations in terms of rules, we rather construct such robust relations using all compatible sets of decision rules.

In Section 2, we recall some basic concepts of DRSA. In Section 3, we discuss an algorithm for generating all compatible MC sets of rules. In Sections 4 and 5, we discuss measures for investigating the robustness of the recommendation suggested by all compatible MC sets of rules. In Section 6 , we account for dealing with incompatibility of preference information. Section 7 proposes how to ensure consistency between the ranking

obtained with the scoring procedures and the provided preference information. Section 8 describes a decision aiding process for the proposed approach. Section 9 concludes the paper.

\section{Notation and Basic Concepts}

We use the following notation:

- $A=\{a, b, \ldots\}$ - a finite set of $n$ alternatives; $B=A \times A$; 
- $G=\left\{g_{1}, g_{2}, \ldots, g_{j}, \ldots, g_{m}\right\}$ - a finite set of $m$ evaluation criteria with ordinal or cardinal scales; for clarity of presentation, we assume that all criteria should be maximized. The set of criteria with the cardinal scale is denoted by $G^{N} \subseteq G$, the set of criteria with the ordinal scale is denoted by $G^{O} \subseteq G$, whereas their respective indices by $\mathcal{J}_{G^{N}}$ and $\mathcal{J}_{G^{O}}\left(G^{N} \cup G^{O}=G\right.$ and $\left.G_{N} \cap G_{O}=\emptyset\right)$. For each $g_{j} \in G^{N}$, the intensity of preference for a pair $a, b \in A$ can be quantified with the performance difference $\Delta_{j}(a, b)=g_{j}(a)-g_{j}(b)$, while for $g_{j} \in G^{O}$ only the order of evaluations $g_{j}(a), g_{j}(b), a, b \in A$, has a meaning.

- $A^{R}=\left\{a^{*}, b^{*}, \ldots\right\}$ - a finite set of reference alternatives, on which the DM accepts to express holistic preferences; we assume that $A^{R} \subseteq A$.

Table A.1 provided in the e-Appendix A (available online) summarizes the notation used throughout the paper.

Dominance relation. When analyzing the performances of alternatives on multiple criteria, alternative $a \in A$ dominates alternative $b \in A$ with respect to a set of criteria $P \subseteq G\left(a D_{P} b\right)$ if and only if $g_{j}(a) \geq g_{j}(b)$ for all $g_{j} \in P$.

Pairwise comparisons. To enrich the dominance relation, we assume the DM provides a set of pairwise comparisons concerning a subset of reference alternatives $A^{R} \subseteq A$. Let us denote the set of pairs of reference alternatives compared by the DM by $B^{R} \subseteq A^{R} \times A^{R}$. For a pair $\left(a^{*}, b^{*}\right)$ of reference alternatives, the DM may state that:

- $a^{*}$ is at least as good as $b^{*}$, being equivalent to $a^{*}$ outranks $b^{*}$, denoted by $a^{*} S b^{*}$ or $\left(a^{*}, b^{*}\right) \in S$; relation $S$ is a weak preference relation, being reflexive;

- $a^{*}$ is not at least as good as $b^{*}$, being equivalent to $a^{*}$ does not outrank $b^{*}$, denoted by $a^{*} S^{c} b^{*}$ or $\left(a^{*}, b^{*}\right) \in S^{c} ;$ relation $S^{c}$ is irreflexive.

In particular, the DM may specify a complete pre-order for the subset of reference alternatives. Subsequently, these statements are automatically translated to the respective outranking and non-outranking relations, i.e., if $a^{*} \succ b^{*}$, then $a^{*} S b^{*}$ and $b^{*} S^{c} a^{*}$.

Following the paradigm of ordinal regression (Jacquet-Lagreze and Siskos, 1982), we assume the DM has some knowledge about the considered alternatives, and thus, that (s)he is able to express preferences by comparing pairwise some reference alternatives relatively well known to this DM. The selection of pairs to be compared does not follow any specific algorithm made in DM's mind; the order of selection may, however, be dependent on the DM's conviction that a pair of alternatives should be compared in a given way. 
The subset of reference alternatives can be composed of real or fictitious alternatives, that refer to some real past decisions of the DM, or to some hypothetical decisions that the DM is generating in order to exemplify her/his preferences (Greco et al., 2011). In any case, the pairs selected for comparison cannot be related by the dominance relation. Only in this way, the expressed preference information may be used to enrich the dominance order following directly from multiple criteria evaluations of alternatives.

\section{Illustrative example (part 1): input data and pairwise comparisons.}

For the purpose of illustration, we consider a real world problem of innovation assessment for a subset of Polish cities. It was originally presented in (Rzeczpospolita, 2012). We consider a set of 16 cities evaluated on six criteria with an increasing direction of preference: incomes (in PLN) per inhabitant $\left(g_{1}\right)$, expenditures (in PLN) per inhabitant $\left(g_{2}\right)$, funds received from the European Union (EU) (in PLN) per inhabitant $\left(g_{3}\right)$, share of the expenditures spent for the non-government organizations (in $\% ; g_{4}$ ), expenditures for housing management (in PLN) per inhabitant $\left(g_{5}\right)$, and expenditures for the city promotion (in $\% ; g_{6}$ ). The performances of cities on the six criteria are presented in Table 1. The objective of the study is to rank the cities with respect to their innovativeness. The preference information consists of the complete order of five reference alternatives:

$$
\text { Poznan }\left(a_{2}\right) \succ \text { Katowice }\left(a_{5}\right) \succ \text { Slupsk }\left(a_{10}\right) \succ \text { Lomza }\left(a_{13}\right) \succ \text { Ostroleka }\left(a_{16}\right) \text {. }
$$

It can be decomposed to 25 pairwise comparisons: 15 corresponding to the truth of the outranking relation $S$ (e.g., $a_{2} S a_{2}$ and $a_{2} S a_{5}$ ), and another 10 to its falsity $S^{c}$ (e.g., $a_{5} S^{c} a_{2}$ ).

\begin{tabular}{rccccccc}
\multicolumn{7}{c}{ Table 1: Cities' performances. } \\
City & Code & $g_{1}$ & $g_{2}$ & $g_{3}$ & $g_{4}$ & $g_{5}$ & $g_{6}$ \\
Gdansk & $a_{1}$ & 4555 & 5566 & 295 & 1.25 & 419 & 0.75 \\
Poznan & $a_{2}$ & 4481 & 5551 & 74 & 2.00 & 340 & 0.65 \\
Wroclaw & $a_{3}$ & 5537 & 5741 & 397 & 1.81 & 385 & 1.02 \\
Bialystok & $a_{4}$ & 4309 & 4977 & 524 & 17.57 & 393 & 0.48 \\
Katowice & $a_{5}$ & 4434 & 4577 & 68 & 1.50 & 694 & 0.55 \\
Cracow & $a_{6}$ & 4412 & 4390 & 131 & 1.36 & 221 & 0.20 \\
Kalisz & $a_{7}$ & 3824 & 4125 & 164 & 1.55 & 623 & 0.38 \\
Opole & $a_{8}$ & 4398 & 4603 & 199 & 0.70 & 174 & 0.22 \\
Jelenia Gora & $a_{9}$ & 4053 & 4802 & 436 & 1.29 & 154 & 0.62 \\
Slupsk & $a_{10}$ & 4318 & 4753 & 212 & 0.51 & 249 & 0.40 \\
Tarnow & $a_{11}$ & 4235 & 4430 & 163 & 1.00 & 202 & 0.19 \\
Gorzow Wlkp. & $a_{12}$ & 3421 & 3771 & 156 & 1.71 & 150 & 0.05 \\
Lomza & $a_{13}$ & 4518 & 4859 & 706 & 0.52 & 37 & 0.21 \\
Zabrze & $a_{14}$ & 3392 & 3434 & 133 & 0.46 & 450 & 0.17 \\
Zory & $a_{15}$ & 3555 & 3552 & 163 & 0.27 & 206 & 0.19 \\
Ostroleka & $a_{16}$ & 4204 & 4353 & 117 & 0.95 & 39 & 0.16
\end{tabular}

Dominance relation for pairs of alternatives. Pair of alternatives $(a, b) \in B^{R}$ dominates pair $(c, d) \in B^{R}$ with respect to a set of criteria $P \subseteq G\left((a, b) D_{P, 2}(c, d)\right)$ if and only if (Greco et al., 1999b, 2001a; Szelag et al., 2013): 
- for all $g_{j} \in G^{N} \cap P, \Delta_{j}(a, b) \geq \Delta_{j}(c, d)$, where $\Delta_{j}(a, b)=g_{j}(a)-g_{j}(b)$;

- for all $g_{j} \in G^{O} \cap P, g_{j}(a) \geq g_{j}(c)$ and $g_{j}(b) \leq g_{j}(d)$.

If $(a, b)$ dominates $(c, d)$ with respect to $G$, it is reasonable to assume that if $c S d$, then $a S b$, and if $a S^{c} b$, then $c S^{c} d$. If this is not the case, an inconsistency with respect to dominance relation for pairs of alternatives in $B^{R}$ occurs (Szelag et al., 2014). It needs to be accounted appropriately at the data structuring phase.

Granules of knowledge. The set $D_{P, 2}^{+}(a, b)$ of pairs of alternatives which dominate $(a, b) \in B^{R}$ is called the P-dominating set (Greco et al., 1999b, 2001a; Szelag et al., 2013):

$$
D_{P, 2}^{+}(a, b)=\left\{(c, d) \in B^{R},(c, d) D_{P, 2}(a, b)\right\}
$$

The set $D_{P, 2}^{-}(a)$ of pairs of alternatives which are dominated by $(a, b)$ is called the $P$-dominated set (Szelag et al., 2013):

$$
D_{P, 2}^{-}(a, b)=\left\{(c, d) \in B^{R},(a, b) D_{P, 2}(c, d)\right\}
$$

Rough approximations. In DRSA, the $P$-dominating and $P$-dominated sets are used for rough approximation of upward and downward unions of preference relations (Greco et al., 1999a). The $P$-lower and $P$-upper approximations of $S$ and $S^{c}$ with respect to $P \subseteq G$, are defined, respectively, as (Greco et al., 1999b, 2001a; Szelag et al., 2013):

$$
\begin{gathered}
\underline{P}(S)=\left\{(a, b) \in B^{R}: D_{P, 2}^{+}(a, b) \subseteq S\right\} \text { and } \bar{P}(S)=\left\{(a, b) \in B^{R}: S \cap D_{P, 2}^{-}(a, b) \neq \emptyset\right\}, \\
\underline{P}\left(S^{c}\right)=\left\{(a, b) \in B^{R}: D_{P, 2}^{-}(a, b) \subseteq S^{c}\right\} \text { and } \bar{P}\left(S^{c}\right)=\left\{(a, b) \in B^{R}: S^{c} \cap D_{P, 2}^{+}(a, b) \neq \emptyset\right\} .
\end{gathered}
$$

Finally, the $P$-boundaries of $S$ and $S^{c}$ are defined as:

$$
B n_{P}(S)=\bar{P}(S) \backslash \underline{P}(S), B n_{P}\left(S^{c}\right)=\bar{P}\left(S^{c}\right) \backslash \underline{P}\left(S^{c}\right),
$$

and they are equal to each other. Intuitively, the lower (upper) approximation contains pairs of alternatives which certainly (possibly) belong to relation $S$ or $S^{c}$.

\section{Illustrative example (part 2): rough approximations of relations $S$ and $S^{c}$.}

The provided 25 pairwise comparisons are consistent, i.e., there is no dominated pair of alternative in $S$ while 
a pair dominating it is in $S^{c}$. The lower approximations of relations $S$ and $S^{c}$ are as follows:

$$
\begin{array}{r}
\underline{P}(S)=\left\{\left(a_{2}, a_{5}\right),\left(a_{2}, a_{10}\right),\left(a_{2}, a_{13}\right),\left(a_{2}, a_{16}\right),\left(a_{5}, a_{10}\right),\left(a_{5}, a_{13}\right),\left(a_{5}, a_{16}\right),\left(a_{10}, a_{13}\right),\left(a_{10}, a_{13}\right),\left(a_{13}, a_{16}\right),\right. \\
\left.\left(a_{2}, a_{2}\right),\left(a_{5}, a_{5}\right),\left(a_{10}, a_{10}\right),\left(a_{13}, a_{13}\right),\left(a_{16}, a_{16}\right)\right\} \\
\underline{P}\left(S^{c}\right)=\left\{\left(a_{5}, a_{2}\right),\left(a_{10}, a_{2}\right),\left(a_{13}, a_{2}\right),\left(a_{16}, a_{2}\right),\left(a_{10}, a_{5}\right),\left(a_{13}, a_{5}\right),\left(a_{16}, a_{5}\right),\left(a_{13}, a_{10}\right),\left(a_{16}, a_{10}\right),\left(a_{16}, a_{13}\right)\right\} .
\end{array}
$$

Obviously, in this case they are equal to the upper approximations, and, thus, $B n_{P=G}(S)=B n_{P=G}\left(S^{c}\right)=\emptyset$.

\section{Generating All Compatible Minimal-Cover Sets of Rules}

The approach presented in this paper represents the pairwise comparisons expressed by the DM with all compatible MC sets of minimal decision rules. On the one hand, the choice of "if ..., then ..." decision rules is motivated by their interpretability and favorable characteristics as an aggregation operator. In particular, they represent a form of dependency between conditions on some criteria and the exhibited decision about preference relation for a pair of alternatives. On the other hand, inferring all compatible instances of a preference model rather than a single instance is a sign of acting with prudence. In this regard, our aim is to provide the DM with the results of applying all equally desirable preference model instances rather than a single instance selected in an arbitrary way.

In what follows, we focus on certain decision rules which are induced from the lower approximations. For a given relation $S\left(S^{c}\right)$, the decision rules induced under hypothesis that pairs of reference alternatives belonging to $\underline{P}(S)\left(\underline{P}\left(S^{c}\right)\right)$ are positive examples and the pairs belonging $\bar{P}\left(S^{c}\right)(\bar{P}(S))$ are negative examples, suggest a certain relation $S\left(S^{c}\right)$. We can distinguish two types of rules (Greco et al., 1999b, 2001a; Szelag et al., 2013):

- "at least" decision rules, suggesting $a S b$, having the following syntax:

$$
\begin{array}{r}
\text { if } \Delta_{j_{1}}(a, b) \geq \delta_{j_{1}} \text { and } \ldots \text { and } \Delta_{j_{p}}(a, b) \geq \delta_{j_{p}} \text { and } \ldots \text { and } g_{j_{p+1}}(a) \geq r_{j_{p+1}} \text { and } g_{j_{p+1}}(b) \leq s_{j_{p+1}} \\
\quad \text { and } \ldots \text { and } g_{j_{z}}(a) \geq r_{j_{z}} \text { and } g_{j_{z}}(b) \leq s_{j_{z}} \text { then } a S b \text {, }
\end{array}
$$

where $\delta_{j_{i}} \in\left\{g_{j_{i}}(c)-g_{j_{i}}(d):(c, d) \in B^{R}\right\} \subseteq \mathbb{R}$, for $j_{j} \in\left\{j_{1}, \ldots, j_{p}\right\} \subseteq \mathcal{J}_{G^{N}}$

- "at most" decision rules, suggesting $a S^{c} b$, having the following syntax:

if $\Delta_{j_{1}}(a, b) \leq \delta_{j_{1}}$ and $\ldots$ and $\Delta_{j_{p}}(a, b) \leq \delta_{j_{p}}$ and $\ldots$ and $g_{j_{p+1}}(a) \leq r_{j_{p+1}}$ and $g_{j_{p+1}}(b) \geq s_{j_{p+1}}$ and $\ldots$ and $g_{j_{z}}(a) \leq r_{j_{z}}$ and $g_{j_{z}}(b) \geq s_{j_{z}}$ then $a S^{c} b$, 
where $\left(r_{j_{i}}, s_{j_{i}}\right) \in\left\{\left(g_{j_{i}}(c), g_{j_{i}}(d)\right):(c, d) \in B^{R}\right\} \subseteq \mathbb{R} \times \mathbb{R}$, for $j_{i} \in\left\{j_{p+1}, \ldots, j_{p_{z}}\right\} \subseteq \mathcal{J}_{G^{O}}$.

Definition 3.1. Decision rule $r_{S}\left(r_{S^{c}}\right)$ suggesting relation $S\left(S^{c}\right)$ is minimal in set $\mathcal{R}$ if there is no other rule in $\mathcal{R}$ that would employ weaker elementary conditions or/and a subset of elementary conditions used in $r_{S}\left(r_{S^{c}}\right)$.

A certain decision rule is compatible with a piece of the preference information provided by the DM because it is supported by some positive examples (pairs of reference alternatives from the lower approximation of a preference relation) and it does not cover any negative example (pair of alternatives from the upper approximation of an opposite preference relation). Thus, any set of certain minimal decision rules covering all pairs of reference alternatives from the lower approximation of $S$ and $S^{c}$ is compatible with the whole preference information provided by the DM.

In this section, we discuss an algorithm for generating all compatible MC sets of rules. It starts with generating an exhaustive set of all minimal decision rules (see Section 3.1), and then finds all minimal covers of pairs of alternatives with Integer Linear Programming (ILP) (see Section 3.2).

\subsection{Generating All Compatible Minimal Rules}

In this subsection, we discuss an algorithm which generates all certain minimal decision rules $\mathcal{R} \frac{P(S)}{\text { all }}$ from $\underline{P}(S)$. Its adaptation for generating all minimal rules $\mathcal{R} \underline{P a l l}^{\left(S^{c}\right)}$ from $\underline{P}\left(S^{c}\right)$ is straightforward.

Firstly, we generate a set $C_{1}$ of all candidate elementary conditions which could be used in the condition part of decision rules (see Algorithm 1 in the e-Appendix B). This set is composed of conditions in form $\left(\Delta_{j}(a, b) \geq g_{j}\left(a_{k}^{*}\right)-g_{j}\left(a_{l}^{*}\right)\right)$ (for cardinal criteria) or $\left(g_{j}(a) \geq g_{j}\left(a_{k}^{*}\right)\right.$ and $\left.g_{j}(b) \leq g_{j}\left(a_{l}^{*}\right)\right)$ (for ordinal criteria) with $\left(a_{k}^{*}, a_{l}^{*}\right) \in \underline{P}(S)$.

Secondly, we generate a set of conjunctions of elementary conditions which cover at least one pair of reference alternatives from $\underline{P}(S)$ (see Algorithm 2 in the e-Appendix B). Naturally, within a single conjunction each criterion can be used at most once. Sets $C_{k}$ are built incrementally, starting with $k=2$, then 3 , etc. The set $C_{k}$ of conjunctions of size $k$ contains only these conjunctions which are supported by at least one pair in $\underline{P}(S)$. When a conjunction of size $k$ does not cover any pair from $\bar{P}\left(S^{c}\right)$, it is neglected in the subsequent stages so that to avoid generating candidate conjunctions which are not minimal.

Thirdly, we need to remove conjunctions which either cover some negative example from $\bar{P}\left(S^{c}\right)$ or are not minimal (see Definition 3.1). The remaining conjunctions constitute condition parts of decision rules with conclusion $a S b$. The procedure for generating all minimal decision rules from $\underline{P}(S)$ is summarized as Algorithm 3 in the e-Appendix B. 


\section{Illustrative example (part 3): all compatible minimal rules.}

The set of all minimal rules $\mathcal{R}_{\text {all }}^{A^{R}}$ induced from the lower approximations of $S$ and $S^{c}$ contains 35 certain rules (18 and 17 rules for $\underline{P}(S)$ and $\underline{P}\left(S^{c}\right)$, respectively). These are listed in Table 2 . Among these, there are 10 rules with just a single condition, 19 rules with two ones, and 6 rules with three conditions.

Table 2: All compatible certain minimal rules induced from the lower approximations of $S$ and $S^{c}$.

"at most" decision rules

$r_{S^{c}}^{1}$

$r_{S^{c}}^{2}$

$r_{S^{c}}^{3}$

$r_{S^{c}}^{4}$

$r_{S^{c}}^{5}$

$r_{S^{c}}^{6}$

$r_{S^{c}}^{7}$

$r_{S^{c}}^{8}$

$r_{S^{c}}^{9}$

$r_{S^{c}}^{10}$

$r_{S^{c}}^{11}$

$r_{S^{c}}^{12}$

$r_{S^{c}}^{13}$

$r_{S^{c}}^{14}$

$r_{S^{c}}^{15}$

$r_{S^{c}}^{16}$

$r_{S^{c}}^{17}$ if $\Delta_{4}(a, b) \leq-0.5$ then $a S^{c} b$

if $\Delta_{5}(a, b) \leq-445.0$ then $a S^{c} b$

if $\Delta_{1}(a, b) \leq-230.0$ then $a S^{c} b$

if $\Delta_{2}(a, b) \leq-400.0$ then $a S^{c} b$

if $\Delta_{6}(a, b) \leq-0.05$ then $a S^{c} b$

if $\Delta_{1}(a, b) \leq-47.0$ and $\Delta_{5}(a, b) \leq 2.0$ then $a S^{c} b$

if $\Delta_{3}(a, b) \leq-6.0$ and $\Delta_{5}(a, b) \leq 2.0$ then $a S^{c} b$

if $\Delta_{2}(a, b) \leq-224.0$ and $\Delta_{5}(a, b) \leq 354.0$ then $a S^{c} b$

if $\Delta_{1}(a, b) \leq 37.0$ and $\Delta_{5}(a, b) \leq-91.0$ then $a S^{c} b$

if $\Delta_{2}(a, b) \leq 282.0$ and $\Delta_{5}(a, b) \leq-91.0$ then $a S^{c} b$

if $\Delta_{4}(a, b) \leq 0.44$ and $\Delta_{5}(a, b) \leq-91.0$ then $a S^{c} b$

if $\Delta_{1}(a, b) \leq-114.0$ and $\Delta_{2}(a, b) \leq-224.0$ then $a S^{c} b$

if $\Delta_{2}(a, b) \leq-224.0$ and $\Delta_{4}(a, b) \leq 0.44$ then $a S^{c} b$

if $\Delta_{1}(a, b) \leq-114.0$ and $\Delta_{3}(a, b) \leq-589.0$ then $a S^{c} b$

if $\Delta_{3}(a, b) \leq-589.0$ and $\Delta_{4}(a, b) \leq 0.44$ then $a S^{c} b$

if $\Delta_{1}(a, b) \leq-47.0$ and $\Delta_{3}(a, b) \leq-589.0$

and $\Delta_{5}(a, b) \leq 354.0$ then $a S^{c} b$

if $\Delta_{2}(a, b) \leq 282.0$ and $\Delta_{3}(a, b) \leq-589.0$

and $\Delta_{5}(a, b) \leq 354.0$ then $a S^{c} b$ "at least" decision rules

$r_{S}^{1} \quad$ if $\Delta_{6}(a, b) \geq 0.0$ then $a S b$

$r_{S}^{2} \quad$ if $\Delta_{4}(a, b) \geq 0.5$ then $a S b$

$r_{S}^{3} \quad$ if $\Delta_{5}(a, b) \geq 445.0$ then $a S b$

$r_{S}^{4} \quad$ if $\Delta_{1}(a, b) \geq 230.0$ then $a S b$

$r_{S}^{5} \quad$ if $\Delta_{2}(a, b) \geq 400.0$ then $a S b$

$r_{S}^{6} \quad$ if $\Delta_{3}(a, b) \geq 0.0$ and $\Delta_{5}(a, b) \geq-2.0$ then $a S b$

$r_{S}^{7} \quad$ if $\Delta_{2}(a, b) \geq 224.0$ and $\Delta_{5}(a, b) \geq-354.0$ then $a S b$

$r_{S}^{8} \quad$ if $\Delta_{4}(a, b) \geq-0.44$ and $\Delta_{5}(a, b) \geq 91.0$ then $a S b$

$r_{S}^{9} \quad$ if $\Delta_{1}(a, b) \geq-37.0$ and $\Delta_{5}(a, b) \geq-2.0$ then $a S b$

$r_{S}^{10} \quad$ if $\Delta_{2}(a, b) \geq-282.0$ and $\Delta_{5}(a, b) \geq-2.0$ then $a S b$

$r_{S}^{11} \quad$ if $\Delta_{1}(a, b) \geq 114.0$ and $\Delta_{2}(a, b) \geq 224.0$ then $a S b$

$r_{S}^{12} \quad$ if $\Delta_{2}(a, b) \geq 224.0$ and $\Delta_{4}(a, b) \geq-0.44$ then $a S b$

$r_{S}^{13} \quad$ if $\Delta_{1}(a, b) \geq 114.0$ and $\Delta_{3}(a, b) \geq 589.0$ then $a S b$

$r_{S}^{14} \quad$ if $\Delta_{3}(a, b) \geq 589.0$ and $\Delta_{4}(a, b) \geq-0.44$ then $a S b$

$r_{S}^{15} \quad$ if $\Delta_{1}(a, b) \geq 47.0$ and $\Delta_{3}(a, b) \geq 589.0$

and $\Delta_{5}(a, b) \geq-354.0$ then $a S b$

$r_{S}^{16} \quad$ if $\Delta_{2}(a, b) \geq-282.0$ and $\Delta_{3}(a, b) \geq 589.0$

and $\Delta_{5}(a, b) \geq-354.0$ then $a S b$

$r_{S}^{17} \quad$ if $\Delta_{1}(a, b) \geq-200.0$ and $\Delta_{4}(a, b) \geq-0.44$

and $\Delta_{5}(a, b) \geq-2.0$ then $a S b$

$r_{S}^{18} \quad$ if $\Delta_{3}(a, b) \geq-494.0$ and $\Delta_{4}(a, b) \geq-0.44$

and $\Delta_{5}(a, b) \geq-2.0$ then $a S b$

\subsection{Generating All Minimal Covers}

We are interested in generating the complete sets of rules, which means that each of these sets is able to cover all pairs of reference alternatives compared by the DM and included in lower approximations of $S$ and $S^{c}$. These sets are fully authorized to pretend that they represent preferences of the DM exhibited through the provided pairwise comparisons. Inviting the DM to make her/his own selection of rules would mean that we require from the DM an extra preference information with respect to selected rules. As the number of all rules can be quite large, this would require a cognitive effort on the part of the DM which is non-acceptable.

In this subsection, we discuss an algorithm which generates all complete MC sets of rules $\mathcal{R} \frac{P}{m}(S)$ for $\underline{P}(S)$ by exploiting all minimal rules $\mathcal{R} \frac{P(S)}{\text { all }}$. In the same way, one can generate all MC sets of rules $\mathcal{R} \frac{P\left(S^{c}\right)}{m c}$ for $\underline{P}\left(S^{c}\right)$.

Definition 3.2. A set $\mathcal{R}$ of certain rules induced from the lower approximations of outranking and nonoutranking relations is complete if each $(a, b) \in \underline{P}(S)$ supports at least one decision rule $r_{S}$ from $\mathcal{R}$ whose 
consequent is aSb and each $(a, b) \in \underline{P}\left(S^{c}\right)$ supports at least one decision rule $r_{S^{c}}$ from $\mathcal{R}$ whose consequent is $a S^{c} b$.

Definition 3.3. A set $\mathcal{R}$ of certain decision rules is minimal-cover iff it is complete and non-redundant, i.e., exclusion of any rule $r$ from $\mathcal{R}$ makes it non-complete.

Finding a MC set of rules $\mathcal{R}^{\underline{P}}(S)$ for $\underline{P}(S)$ is analogous to solving the minimum set cover problem (Vazirani, 2001). To construct it, we need to solve a dedicated ILP problem:

$$
\text { Minimize : } f_{w}=\sum_{r_{k} \in \mathcal{R} \frac{P}{\text { all }}(S)} v\left(r_{k}\right)
$$

$$
\left.\begin{array}{ll}
\text { s.t. } & \sum_{r_{k} \in \mathcal{R}} \underline{P l l}_{\text {all }}^{(S)} \text { covering }\left(a_{s}, a_{l}\right) \\
& v\left(r_{k}\right) \in\{0,1\}, \text { for all } r_{k} \in \mathcal{R}{ }_{\text {all }}^{P} .(S)
\end{array}\right\} E_{\text {cover }}^{\text {minimal }}
$$

If $v\left(r_{k}\right)=1, r_{k} \in \mathcal{R} \frac{P}{\text { all }}(S)$ is included in the set of rules $\mathcal{R} \underline{P}^{(S)}$ covering all pairs in $\underline{P}(S)$, where $w$ is an index of iteration, because the above procedure will be repeated as many times as there are feasible solutions of problem (7). The optimal solution of the above ILP (denoted by ${ }^{*} ;$ e.g., $f_{w}^{*}$ and $\left[v\left(r_{k}\right)^{*}, r_{k} \in \mathcal{R} \frac{P(S)}{a l l}\right]$ ) indicates the first MC set of rules:

$$
\mathcal{R} \frac{P(S)}{w=1}=\left\{r_{k} \in \mathcal{R} \frac{P}{\text { all }}(S), \text { such that } v\left(r_{k}\right)^{*}=1\right\}
$$

However, there may exist other MC sets of rules in $\mathcal{R} \frac{P(S)}{m} r c$. They can be identified within an iterative procedure. For this purpose, in each iteration $w=2, \ldots,\left|\mathcal{R} \frac{P}{m} r c\right|$, we need to solve problem (7) while forbidding finding again the same solutions as found in previous iterations $(w-1, w-2, \ldots, 1)$. This can be achieved by adding the following constraints on the sum of respective binary variables, for $z=1, \ldots, w-1$ :

$$
\sum_{r_{k} \in \mathcal{R}_{\bar{z}}^{\frac{P}{(S)}}} v\left(r_{k}\right) \leq f_{z}^{*}-1
$$

Finally, all compatible MC sets of rules $\mathcal{R}^{A^{R}}$ are formed by the following product:

$$
\mathcal{R}^{A^{R}}=\mathcal{R} \frac{P}{m}(S) \times \mathcal{R} \frac{P}{m r c}\left(S^{c}\right)
$$

\section{Illustrative example (part 4): all compatible sets of rules.}

Using rules from Table 2 as an input, we generate all MC sets of rules for the lower approximations of relations $S$ and $S^{c}$. They are presented in Table 3. There are 23 and 24 MC sets of rules for pairs of reference alternatives from $\underline{P}\left(S^{c}\right)$ and $\underline{P}(S)$, respectively. As indicated by Equation (10), combination of these minimal 
rule covers leads to $552 \mathrm{MC}$ sets of minimal rules $\mathcal{R}^{A^{R}}$ which reproduce all outranking and non-outranking relations derived from the pairwise comparisons provided by the DM. The number of rules in each of these sets ranges from 2 (in case $r_{S}^{1}$ and $r_{S^{c}}^{5}$ are used) to 6 (e.g., $\left\{r_{S}^{7}, r_{S}^{8}, r_{S}^{9}, r_{S^{c}}^{1}, r_{S^{c}}^{11}, r_{S^{c}}^{14}\right\}$ ).

Table 3: All MC sets of rules for the lower approximations of relations $S$ and $S^{c}$

$\mathcal{R} \frac{P}{m}{ }_{r c}^{(S)}=24$ minimal sets of rules covering pairs of reference alternatives from $\underline{P}(S)$

$\mathcal{R}_{1}^{\underline{P}(S)}=\left\{r_{S}^{1}\right\}, \mathcal{R}_{2}^{\underline{P}(S)}=\left\{r_{S}^{2}, r_{S}^{18}\right\}, \mathcal{R}_{3}^{\underline{P}(S)}=\left\{r_{S}^{2}, r_{S}^{10}\right\}, \mathcal{R}_{4}^{\underline{P}(S)}=\left\{r_{S}^{5}, r_{S}^{10}\right\}, \mathcal{R}_{5}^{\underline{P}(S)}=\left\{r_{S}^{5}, r_{S}^{17}\right\}, \mathcal{R}_{6}^{\underline{P}(S)}=\left\{r_{S}^{2}, r_{S}^{17}\right\}$,

$\mathcal{R}_{7}^{P}(S)=\left\{r_{S}^{12}, r_{S}^{17}\right\}, \mathcal{R}_{8}^{P(S)}=\left\{r_{S}^{10}, r_{S}^{12}\right\}, \mathcal{R}_{9}^{\underline{P}(S)}=\left\{r_{S}^{7}, r_{S}^{10}\right\}, \mathcal{R}_{10}^{P(S)}=\left\{r_{S}^{7}, r_{S}^{17}\right\}, \mathcal{R}_{11}^{P(S)}=\left\{r_{S}^{3}, r_{S}^{5}, r_{S}^{18}\right\}, \mathcal{R}_{12}^{P(S)}=\left\{r_{S}^{5}, r_{S}^{8}, r_{S}^{18}\right\}$,

$\mathcal{R}_{13}^{P}(S)=\left\{r_{S}^{3}, r_{S}^{12}, r_{S}^{18}\right\}, \mathcal{R}_{14}^{P(S)}=\left\{r_{S}^{8}, r_{S}^{12}, r_{S}^{18}\right\}, \mathcal{R}_{15}^{P(S)}=\left\{r_{S}^{3}, r_{S}^{7}, r_{S}^{18}\right\}, \mathcal{R} \frac{P(S)}{16}=\left\{r_{S}^{7}, r_{S}^{8}, r_{S}^{18}\right\}, \mathcal{R}_{17}^{P}(S)=\left\{r_{S}^{5}, r_{S}^{6}, r_{S}^{8}\right\}$,

$\mathcal{R}_{18}^{P}(S)=\left\{r_{S}^{5}, r_{S}^{8}, r_{S}^{9}\right\}, \mathcal{R} \frac{P}{19}(S)=\left\{r_{S}^{2}, r_{S}^{6}, r_{S}^{8}\right\}, \mathcal{R} \frac{P}{20}(S)=\left\{r_{S}^{2}, r_{S}^{8}, r_{S}^{9}\right\}, \mathcal{R} \frac{P}{21}(S)=\left\{r_{S}^{6}, r_{S}^{8}, r_{S}^{12}\right\}, \mathcal{R} \frac{P}{22}(S)=\left\{r_{S}^{8}, r_{S}^{9}, r_{S}^{12}\right\}$,

$\mathcal{R} \frac{P}{23}(S)=\left\{r_{S}^{6}, r_{S}^{7}, r_{S}^{8}\right\}, \mathcal{R} \frac{P}{24}(S)=\left\{r_{S}^{7}, r_{S}^{8}, r_{S}^{9}\right\}$

$\mathcal{R} \frac{P}{m}\left(S^{c}\right)=23$ minimal sets of rules covering pairs of reference alternatives from $\underline{P}\left(S^{c}\right)$

$\mathcal{R}_{1}^{\underline{P}\left(S^{c}\right)}=\left\{r_{S^{c}}^{5}\right\}, \mathcal{R}_{\frac{P}{2}}^{\left(S^{c}\right)}=\left\{r_{S^{c}}^{10}, r_{S^{c}}^{13}\right\}, \mathcal{R}_{3}^{\underline{P}\left(S^{c}\right)}=\left\{r_{S^{c}}^{8}, r_{S^{c}}^{10}\right\}, \mathcal{R}_{4}^{\underline{P}\left(S^{c}\right)}=\left\{r_{S^{c}}^{11}, r_{S^{c}}^{13}\right\}, \mathcal{R}_{5}^{\frac{P}{\left(S^{c}\right)}}=\left\{r_{S^{c}}^{8}, r_{S^{c}}^{11}\right\}, \mathcal{R}_{6}^{\underline{P}\left(S^{c}\right)}=\left\{r_{S^{c}}^{4}, r_{S^{c}}^{11}\right\}$, $\mathcal{R}_{7}^{P}\left(S^{c}\right)=\left\{r_{S^{c}}^{4}, r_{S^{c}}^{10}\right\}, \mathcal{R}_{8}^{P(S)}=\left\{r_{S^{c}}^{1}, r_{S^{c}}^{10}, r_{S^{c}}^{15}\right\}, \mathcal{R}_{9}^{\left(S^{c}\right)}=\left\{r_{S^{c}}^{1}, r_{S^{c}}^{11}, r_{S^{c}}^{15}\right\}, \mathcal{R}_{10}^{P\left(S^{c}\right)}=\left\{r_{S^{c}}^{1}, r_{S^{c}}^{10}, r_{S^{c}}^{17}\right\}, \mathcal{R}_{11}^{P}\left(S^{c}\right)=\left\{r_{S^{c}}^{1}, r_{S^{c}}^{11}, r_{S^{c}}^{17}\right\}$, $\mathcal{R}_{12}^{P}\left(S^{c}\right)=\left\{r_{S^{c}}^{1}, r_{S^{c}}^{7}, r_{S^{c}}^{10}\right\}, \mathcal{R} \frac{P}{13}{ }^{\left(S^{c}\right)}=\left\{r_{S^{c}}^{1}, r_{S^{c}}^{7}, r_{S^{c}}^{11}\right\}, \mathcal{R} \frac{P}{14} S^{\left(S^{c}\right)}=\left\{r_{S^{c}}^{1}, r_{S^{c}}^{10}, r_{S^{c}}^{16}\right\}, \mathcal{R} \frac{P}{15}\left(S^{c}\right)=\left\{r_{S^{c}}^{1}, r_{S^{c}}^{11}, r_{S^{c}}^{16}\right\}, \mathcal{R}_{16}^{P\left(S^{c}\right)}=\left\{r_{S^{c}}^{1}, r_{S^{c}}^{3}, r_{S^{c}}^{10}\right\}$, $\left.\mathcal{R}_{17}^{P}\left(S^{c}\right)=\left\{r_{S^{c}}^{1}, r_{S^{c}}^{3}, r_{S^{c}}^{11}\right\}, \mathcal{R} \frac{P}{18} S^{c}\right)=\left\{r_{S^{c}}^{1}, r_{S^{c}}^{6}, r_{S^{c}}^{10}\right\}, \mathcal{R}_{19}^{\underline{P}\left(S^{c}\right)}=\left\{r_{S^{c}}^{1}, r_{S^{c}}^{6}, r_{S^{c}}^{11}\right\}, \mathcal{R}_{20}^{\underline{P}\left(S^{c}\right)}=\left\{r_{S^{c}}^{1}, r_{S^{c}}^{10}, r_{S^{c}}^{12}\right\}, \mathcal{R}_{21}^{\underline{P}\left(S^{c}\right)}=\left\{r_{S^{c}}^{1}, r_{S^{c}}^{11}, r_{S^{c}}^{12}\right\}$, $\mathcal{R} \frac{P}{22}\left(S^{c}\right)=\left\{r_{S^{c}}^{1}, r_{S^{c}}^{10}, r_{S^{c}}^{14}\right\}, \mathcal{R} \frac{P}{23}\left(S^{c}\right)=\left\{r_{S^{c}}^{1}, r_{S^{c}}^{11}, r_{S^{c}}^{14}\right\}$

\section{Robustness Analysis for Outranking and Non-outranking Relations}

Robustness analysis accounts for the inaccuracy, uncertainty, and biases observed in the actual decision support processes. In our case, the reasons underlying these imperfections include the incompleteness of pairwise comparisons of reference alternatives (as opposed to a complete ranking of all alternatives) and indetermination in the definition of a single instance of the preference model while there exist many compatible MC rule sets (as opposed to an arbitrary selection of a single set of rules). As noted by Kadziński and Tervonen (2013), investigation of the robustness of the provided conclusions consists in verifying whether they are valid for all or for the most plausible instances of an assumed preference model.

To yield a specific preference relation in the set of alternatives, for each pair $(a, b) \in B$, we account for both the positive and negative evidence. The previous is provided by the decision rules with conclusion $a S b$, whereas the latter is raised by rules supporting $a S^{c} b$. In this section, we discuss how to investigate the robustness of the preference relations with respect to all compatible MC sets of rules. In particular, the robust recommendations are materialized with the necessary relation, as well as with outranking and non-outranking acceptability indices.

\subsection{Two Valued Logic}

Given set $\mathcal{R}^{A^{R}}$ of compatible minimal cover sets of rules, for each pair of alternatives $(a, b) \in B$, we may analyze the suggested recommendation in terms of the necessary and the possible (see, e.g., (Greco et al., $2008,2011)$ ). In this case, the necessary and the possible concern both $S$ and $S^{c}$. Then, analogously to (Greco 
et al., 2011), one obtains a pair of outranking (non-outranking) relations on $B$ characterized by different levels of certainty, such that for any $(a, b) \in B$ :

- a necessarily outranks $b\left(a S^{N} b\right)$ if $a$ outranks $b$ (a necessarily does not outrank $b\left(a S^{c N} b\right)$ if $a$ does not outrank $b$ ) for all compatible sets of rules, i.e.:

$$
a S^{N} b \Leftrightarrow \forall \mathcal{R} \in \mathcal{R}^{A^{R}}: a S b \quad\left(a S^{c N} b \Leftrightarrow \forall \mathcal{R} \in \mathcal{R}^{A^{R}}: a S^{c} b\right)
$$

- a possibly outranks $b\left(a S^{P} b\right)$ if $a$ outranks $b$ (a possibly does not outrank $b\left(a S^{c P} b\right)$ if $a$ does not outrank b) for at least one compatible set of rules, i.e.:

$$
a S^{P} b \Leftrightarrow \exists \mathcal{R} \in \mathcal{R}^{A^{R}}: a S b \quad\left(a S^{c P} b \Leftrightarrow \exists \mathcal{R} \in \mathcal{R}^{A^{R}}: a S^{c} b\right)
$$

Since all compatible sets of rules are known, for each of them we can verify whether for $(a, b) \in B$ it suggests $a S b$ or $a S^{c} b$. Let us denote the sets of rules from $\mathcal{R}^{A^{R}}$ suggesting $a S b$ or $a S^{c} b$ by, respectively, $\mathcal{R}_{a S b} \subseteq \mathcal{R}^{A^{R}}$ and $\mathcal{R}_{a S^{c} b} \subseteq \mathcal{R}^{A^{R}}$. Their cardinalities can be used to derive pairwise outranking $\operatorname{POI}(a, b)$ and non-outranking $\operatorname{PNOI}(a, b)$ indices defined as the shares of compatible sets of rules from $\mathcal{R}^{A^{R}}$ for which $a$ outranks or does not outrank $b$, respectively. Consequently, for $(a, b) \in B, \operatorname{POI}(a, b)$ and $\operatorname{PNOI}(a, b)$, both belonging to interval $[0,1]$, can be computed as:

$$
\operatorname{POI}(a, b)=\frac{\left|\mathcal{R}_{a S b}\right|}{\left|\mathcal{R}^{A^{R}}\right|} \text { and } \operatorname{PNOI}(a, b)=\frac{\left|\mathcal{R}_{a S^{c} b}\right|}{\left|\mathcal{R}^{A^{R}}\right|}
$$

The pairwise outranking and non-outranking indices can be interpreted as estimates of a probability of, respectively, outranking and non-outranking relations for an ordered pair of alternatives (Kadziński and Tervonen, 2013). In this way, their main role is to indicate how often the possible (though non-necessary) results are observed in case of the indetermination of the preference model caused by consideration of all compatible MC sets of rules instead of a single model being one MC set of rules.

If these indices are positive, then the respective possible preference relation holds, whereas in case they are equal to one, the necessary relations is true. Since a single compatible MC set of rules may suggest for a pair $(a, b) \in B$, both outranking and non-outranking, or either one of these relations, or none of them, the following proposition holds.

Proposition 4.1. For $(a, b) \in B, 0 \leq \operatorname{POI}(a, b)+\operatorname{PNOI}(a, b) \leq 2$. 


\section{Illustrative example (part 5): possible and necessary outranking relations and outranking in- dices.}

Let us examine the variety of the recommendations proposed by all compatible MC sets of rules for the 16 alternatives (i.e., 256 ordered pairs of alternatives) provided in Table 1. The POIs and PNOIs are presented, respectively, in Tables 4 and 5 . For example, for $\left(a_{4}, a_{1}\right) \in A \times A$ :

- $\mathcal{R}_{a_{4} S a_{1}}$ includes all sets of rules from $\mathcal{R}^{A^{R}}$, that are composed of one of the following subsets $\mathcal{R}_{2} \frac{P(S)}{}$, $\mathcal{R} \frac{P}{3}{ }^{(S)}, \mathcal{R} \frac{P}{6}{ }^{(S)}, \mathcal{R} \frac{P}{19}{ }^{(S)}$, or $\mathcal{R} \frac{P}{20}^{(S)}$, containing rules covering pair $\left(a_{4}, a_{1}\right)$ and suggesting $a_{4} S a_{1}$, combined with any subset of rules from family $\mathcal{R}_{m r c}^{P\left(S^{c}\right)}$; overall, since $\left|\mathcal{R} \frac{P}{m r c}\left(^{c}\right)\right|=23$, family $\mathcal{R}_{a_{4} S a_{1}}$ is composed of $5 \cdot 23=115$ different sets of rules;

- $\mathcal{R}_{a_{4} S^{c} a_{1}}$ includes all sets of rules from $\mathcal{R}^{A^{R}}$, that are composed of one of the following subsets $\mathcal{R}_{1}^{\underline{P}\left(S^{c}\right)}$,

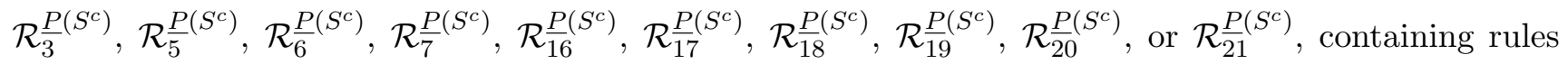
covering pair $\left(a_{4}, a_{1}\right)$ and suggesting $a_{4} S^{c} a_{1}$, combined with any subset of rules from family $\mathcal{R}_{m r c}^{P(S)}$; overall, since $\left|\mathcal{R} \frac{P}{m r c}\right|=24$, family $\mathcal{R}_{a_{4} S^{c} a_{1}}$ is composed of $11 \cdot 24=264$ different sets of rules.

Thus, $\operatorname{POI}\left(a_{4}, a_{1}\right)=\left|\mathcal{R}_{a_{4} S a_{1}}\right| /\left|\mathcal{R}^{A^{R}}\right|=(5 \cdot 23) /(24 \cdot 23)=0.21$ and $\operatorname{PNOI}\left(a_{4}, a_{1}\right)=\left|\mathcal{R}_{a_{4} S^{c} a_{1}}\right| /\left|\mathcal{R}^{A^{R}}\right|=$ $(11 \cdot 24) /(23 \cdot 24)=0.48$.

Analysis of outranking indices reveals that the method restores the DM's preference information, e.g.:

$$
a_{2} \succ a_{5} \Rightarrow a_{2} S^{N} a_{5} \text { and } \operatorname{not}\left(a_{5} S^{P} a_{2}\right) \text { and } a_{5} S^{c N} a_{2} \text { and } \operatorname{not}\left(a_{2} S^{c P} a_{5}\right)
$$

Obviously, the matrices of possible outranking $\left(S^{P}\right)$ and non-outranking $\left(S^{c P}\right)$ relations are more dense than the corresponding matrices of the necessary relations $S^{N}$ and $S^{c N}$. In particular:

- there are 196 pairs of alternatives $(a, b) \in B$, such that $a S^{P} b$; for the remaining 60 ones, $\operatorname{not}\left(a S^{P} b\right)$;

- only for 83 pairs $(a, b) \in B$ the positive evidence is provided by all compatible MC sets of rules (then, $\left.a S^{N} b\right)$

- there are 184 pairs of alternatives $(a, b) \in B$, such that $a S^{c P} b$; for the remaining 82 ones, $\operatorname{not}\left(a S^{c P} b\right)$;

- the possible truth of non-outranking turns out to be the necessary one $\left(S^{c N}\right)$ only for 59 pairs $(a, b) \in B$.

The matrices of POIs and PNOIs supply the following information:

- the most certain recommendation supported either by all compatible MC sets of rules, which concern pairs $(a, b)$ such that $a S^{N} b$ (e.g., $\left.\left(a_{1}, a_{10}\right)\right)$ and $a S^{c N} b$ (e.g., $\left.\left(a_{8}, a_{5}\right)\right)$, or none of these sets (in this case, $\operatorname{not}\left(a S^{P} b\right)\left(\right.$ e.g., $\left.\left(a_{6}, a_{4}\right)\right)$ and $\operatorname{not}\left(S^{c P} b\right)\left(\right.$ e.g, $\left.\left.\left(a_{10}, a_{11}\right)\right)\right)$; 
- uncertainty with respect to $S$ which concerns pairs $(a, b)$ such that $a S^{P} b$ but not $\left(a S^{N} b\right)$ (e.g., $\left.\left(a_{9}, a_{6}\right)\right)$ or to $S^{c}$ concerning pairs $(a, b)$ such that $a S^{c P} b$ but $\operatorname{not}\left(a S^{c N} b\right)$ (e.g., $\left.\left(a_{1}, a_{3}\right)\right)$;

- degree of confirmation of the possible relation, not being the necessary one, with respect to the share of compatible sets of rules that confirm it; in particular, this confirmation may be very strong (e.g., $\left.\operatorname{POI}\left(a_{4}, a_{9}\right)=0.96, \operatorname{PNOI}\left(a_{8}, a_{7}\right)=0.87\right)$ or very weak (e.g., $\operatorname{POI}\left(a_{15}, a_{14}\right)=0.04, \operatorname{PNOI}\left(a_{11}, a_{6}\right)=$ $0.11)$.

When analyzing the indices for each (unordered) pair of alternatives $a, b \in A$, one can easily identify pairs for which:

- the advantage of one alternative over the other is undeniable, e.g., $a_{1}$ and $a_{10}$, such that $a_{1} S^{N} a_{10}$, $\operatorname{not}\left(a_{10} S^{P} a_{1}\right), a_{10} S^{c N} a_{1}$, and $\operatorname{not}\left(a_{1} S^{c P} a_{10}\right) ;$

- there exists a hesitation in the statement of superiority of one alternative over the other, e.g., $a_{3}$ and $a_{4}$, such that $a_{3} S^{P} a_{4}, a_{4} S^{P} a_{3}, a_{3} S^{c P} a_{4}$, and $a_{4} S^{c P} a_{3}$.

Table 4: Pairwise outranking indices POIs.

\begin{tabular}{|c|c|c|c|c|c|c|c|c|c|c|c|c|c|c|c|c|}
\hline & $a_{1}$ & $a_{2}$ & $a_{3}$ & $a_{4}$ & $a_{5}$ & $a_{6}$ & $a_{7}$ & $a_{8}$ & $a_{9}$ & $a_{10}$ & $a_{11}$ & $a_{12}$ & $a_{13}$ & $a_{14}$ & $a_{15}$ & $a_{16}$ \\
\hline$a_{1}$ & 1.00 & 0.54 & 0.17 & 0.71 & 0.79 & 1.00 & 0.79 & 1.00 & 1.00 & 1.00 & 1.00 & 0.79 & 1.00 & 1.00 & 1.00 & 1.00 \\
\hline$a_{2}$ & 0.21 & 1.00 & 0.00 & 0.54 & 1.00 & 1.00 & 0.79 & 1.00 & 1.00 & 1.00 & 1.00 & 1.00 & 1.00 & 1.00 & 1.00 & 1.00 \\
\hline$a_{3}$ & 0.25 & 1.00 & 1.00 & 0.54 & 0.79 & 1.00 & 0.79 & 1.00 & 1.00 & 1.00 & 1.00 & 1.00 & 1.00 & 1.00 & 1.00 & 1.00 \\
\hline$a_{4}$ & 0.21 & 0.71 & 0.58 & 1.00 & 0.96 & 1.00 & 1.00 & 1.00 & 0.96 & 1.00 & 1.00 & 1.00 & 0.88 & 1.00 & 1.00 & 1.00 \\
\hline$a_{5}$ & 0.79 & 0.00 & 0.63 & 0.21 & 1.00 & 1.00 & 0.96 & 1.00 & 0.96 & 1.00 & 1.00 & 1.00 & 1.00 & 1.00 & 1.00 & 1.00 \\
\hline$a_{6}$ & 0.00 & 0.00 & 0.00 & 0.00 & 0.00 & 1.00 & 0.25 & 0.83 & 0.63 & 0.21 & 0.83 & 0.96 & 0.71 & 1.00 & 1.00 & 1.00 \\
\hline$a_{7}$ & 0.63 & 0.17 & 0.63 & 0.00 & 0.00 & 0.83 & 1.00 & 0.75 & 0.63 & 0.71 & 0.75 & 1.00 & 0.75 & 1.00 & 1.00 & 0.88 \\
\hline$a_{8}$ & 0.00 & 0.00 & 0.00 & 0.00 & 0.00 & 0.04 & 0.25 & 1.00 & 0.33 & 0.00 & 0.04 & 0.79 & 0.83 & 0.79 & 0.79 & 1.00 \\
\hline$a_{9}$ & 0.00 & 0.00 & 0.00 & 0.04 & 0.29 & 0.79 & 0.54 & 0.25 & 1.00 & 0.25 & 0.54 & 1.00 & 0.88 & 1.00 & 1.00 & 1.00 \\
\hline$a_{10}$ & 0.00 & 0.00 & 0.00 & 0.00 & 0.00 & 0.54 & 0.29 & 0.83 & 0.33 & 1.00 & 0.67 & 0.79 & 1.00 & 0.79 & 1.00 & 1.00 \\
\hline$a_{11}$ & 0.00 & 0.00 & 0.00 & 0.00 & 0.00 & 0.00 & 0.00 & 0.63 & 0.63 & 0.00 & 1.00 & 0.79 & 0.46 & 1.00 & 1.00 & 1.00 \\
\hline$a_{12}$ & 0.00 & 0.00 & 0.00 & 0.00 & 0.00 & 0.00 & 0.00 & 0.21 & 0.00 & 0.21 & 0.21 & 1.00 & 0.58 & 0.71 & 0.21 & 0.71 \\
\hline$a_{13}$ & 0.00 & 0.00 & 0.00 & 0.00 & 0.00 & 0.54 & 0.25 & 0.50 & 0.00 & 0.00 & 0.54 & 0.54 & 1.00 & 0.54 & 0.79 & 1.00 \\
\hline$a_{14}$ & 0.00 & 0.17 & 0.00 & 0.00 & 0.00 & 0.17 & 0.00 & 0.63 & 0.00 & 0.63 & 0.00 & 0.21 & 0.46 & 1.00 & 0.96 & 0.21 \\
\hline$a_{15}$ & 0.00 & 0.00 & 0.00 & 0.00 & 0.00 & 0.00 & 0.00 & 0.29 & 0.00 & 0.00 & 0.21 & 0.54 & 0.46 & 0.04 & 1.00 & 0.21 \\
\hline$a_{16}$ & 0.00 & 0.00 & 0.00 & 0.00 & 0.00 & 0.00 & 0.00 & 0.00 & 0.00 & 0.00 & 0.00 & 0.54 & 0.00 & 0.50 & 0.96 & 1.00 \\
\hline
\end{tabular}

\subsection{Four Valued Logic}

When referring to the recommendation suggested by a single set of rules in terms of $S$ and $S^{c}$, four situations for the outranking are possible (Tsoukias and Vincke, 1995):

(1) true outranking, $a S^{\mathcal{T}} b$, iff $a S b$ and $\operatorname{not}\left(a S^{c} b\right)$;

(2) false outranking, $a S^{\mathcal{F}} b$, iff $a S^{c} b$ and $\operatorname{not}(a S b)$;

(3) unknown outranking, $a S^{\mathcal{U}} b$, iff $\operatorname{not}(a S b)$ and $\operatorname{not}\left(a S^{c} b\right)$; 
Table 5: Pairwise non-outranking indices PNOIs.

\begin{tabular}{|c|c|c|c|c|c|c|c|c|c|c|c|c|c|c|c|c|}
\hline & $a_{1}$ & $a_{2}$ & $a_{3}$ & $a_{4}$ & $a_{5}$ & $a_{6}$ & $a_{7}$ & $a_{8}$ & $a_{9}$ & $a_{10}$ & $a_{11}$ & $a_{12}$ & $a_{13}$ & $a_{14}$ & $a_{15}$ & $a_{16}$ \\
\hline$a_{3}$ & 0.00 & 0.00 & 0.00 & 0.70 & 0.48 & 0.00 & 0.48 & 0.00 & 0.00 & 0.00 & 0.00 & 0.00 & 0.00 & 0.00 & 0.00 & 0.00 \\
\hline$a_{5}$ & 0.39 & 1.00 & 0.48 & 0.96 & 0.00 & 0.00 & 0.00 & 0.00 & 0.13 & 0.00 & 0.00 & 0.00 & 0.00 & 0.00 & 0.00 & 0.00 \\
\hline$a_{6}$ & 1.00 & 1.00 & 1.00 & 1.00 & 1.00 & 0.00 & 1.00 & 0.00 & 0.30 & 0.22 & 0.00 & 0.00 & 0.17 & 0.00 & 0.00 & 0.00 \\
\hline$a_{7}$ & 0.48 & 0.48 & 0.48 & 1.00 & 0.57 & 0.26 & 0.00 & 0.26 & 0.30 & 0.26 & 0.17 & 0.00 & 0.26 & 0.00 & 0.00 & 0.17 \\
\hline$a_{10}$ & 1.00 & 1.00 & 1.00 & 1.00 & 1.00 & 0.70 & 0.83 & 0.00 & 0.74 & 0.00 & 0.00 & 0.70 & 0.00 & 0.48 & 0.00 & 0.00 \\
\hline$a_{11}$ & 1.00 & 1.00 & 1.00 & 1.00 & 1.00 & 0.09 & 0.87 & 0.00 & 0.22 & 0.30 & 0.00 & 0.70 & 0.35 & 0.00 & 0.00 & 0.00 \\
\hline$a_{12}$ & 0.78 & 1.00 & 1.00 & 1.00 & 1.00 & 0.57 & 1.00 & 0.57 & 0.65 & 0.78 & 0.57 & 0.00 & 0.39 & 0.04 & 0.22 & 0.39 \\
\hline$a_{13}$ & 1.00 & 1.00 & 1.00 & 1.00 & 1.00 & 0.83 & 0.87 & 0.96 & 1.00 & 1.00 & 0.48 & 0.83 & 0.00 & 0.48 & 0.48 & 0.00 \\
\hline$a_{14}$ & 1.00 & 1.00 & 1.00 & 1.00 & 1.00 & 0.96 & 1.00 & 0.48 & 1.00 & 0.48 & 0.96 & 0.87 & 0.35 & 0.00 & 0.00 & 0.35 \\
\hline
\end{tabular}

(4) contradictory outranking, $a S^{\mathcal{K}} b$, iff $a S b$ and $a S^{c} b$.

When analyzing robustness of the above four values of outranking, we may again refer to the necessary and the possible. For brevity, we provide explicit definitions referring only to the true outranking $S^{\mathcal{T}}$ :

- the necessary true outranking $S^{\mathcal{T} N}$ is verified for a pair $(a, b) \in B$ iff $\forall \mathcal{R} \in \mathcal{R}^{A^{R}}$ for which $a S^{\mathcal{T}} b$;

- the possible true outranking $S^{\mathcal{T} P}$ is verified for a pair $(a, b) \in B$ iff $\exists \mathcal{R} \in \mathcal{R}^{A^{R}}$ for which $a S^{\mathcal{T}} b$.

When considering the remaining three relations, the definitions for $S^{\mathcal{F} N}, S^{\mathcal{F} P}, S^{\mathcal{U} N}, S^{\mathcal{U} P}, S^{\mathcal{K} N}$, and $S^{\mathcal{K} P}$ can be formulated analogously.

Knowing the necessary and possible outranking and non-outranking relations, we may indicate the truth of the necessary true, false, unknown, and contradictory outranking as follows:

$$
\begin{gathered}
a S^{N} b \text { and } \operatorname{not}\left(a S^{c P} b\right) \Leftrightarrow a S^{\mathcal{T} N} b ; \quad a S^{c N} b \text { and } \operatorname{not}\left(a S^{P} b\right) \Leftrightarrow a S^{\mathcal{F} N} b ; \\
\operatorname{not}\left(a S^{P} b\right) \text { and } \operatorname{not}\left(a S^{c P} b\right) \Leftrightarrow a S^{\mathcal{U N}} b, \quad a S^{N} b \text { and }\left(a S^{c N} b\right) \Leftrightarrow a S^{\mathcal{K N}} b .
\end{gathered}
$$

Let $\operatorname{PTOI}(a, b), \operatorname{PFOI}(a, b), \operatorname{PUOI}(a, b)$, and $\operatorname{PKOI}(a, b)$ be outranking indices representing the share of compatible MC sets of rules suggesting, respectively, $a S^{\mathcal{T}} b, a S^{\mathcal{F}} b, a S^{\mathcal{U}} b$, and $a S^{\mathcal{K}} b$. Since these four relations are mutually exclusive, the following proposition holds.

Proposition 4.2. For each $(a, b) \in B, \operatorname{PTOI}(a, b)+\operatorname{PFOI}(a, b)+\operatorname{PUOI}(a, b)+\operatorname{PKOI}(a, b)=1$.

Different types of outranking relation suggested by all MC sets of rules for a pair $(a, b) \in B$ can be presented graphically using a single point located within a regular tetrahedron. Formally, this representation involves barycentric coordinates on a tetrahedron. The vertices of the tetrahedron correspond to the necessary 
outranking relations $S^{\mathcal{T} N}, S^{\mathcal{U} N}, S^{\mathcal{K} N}$, and $S^{\mathcal{F} N}$ (see Figure 1). Then, each of the indices PTOI $(a, b)$, PUOI $(a, b), \operatorname{PKOI}(a, b)$, and PFOI $(a, b)$ determines the distance of the surface on which the point is located from the vertex $S^{\mathcal{T} N}, S^{\mathcal{U} N}, S^{\mathcal{K} N}$, and $S^{\mathcal{F} P}$, respectively. For example, if $\operatorname{PTOI}(a, b)=1.0$, then the point is located in the vertex $S^{\mathcal{T} N}$; if $\operatorname{PTOI}(a, b)=0.0$, it is located on the surface determined by vertices $S^{\mathcal{U} N}$, $S^{\mathcal{K} N}$, and $S^{\mathcal{F} N}$; if $\operatorname{PTOI}(a, b) \in(0.0,1.0)$, the point is located on the surface parallel to the one delimited by $S^{\mathcal{U} N}, S^{\mathcal{K} N}$, and $S^{\mathcal{F} N}$ whose distance from $S^{\mathcal{T} N}$ is proportional to $\operatorname{PTOI}(a, b)$ (i.e., the greater $\operatorname{PTOI}(a, b)$, the less the distance). Such representation for four exemplary pairs of alternatives is illustrated in Figure 1.
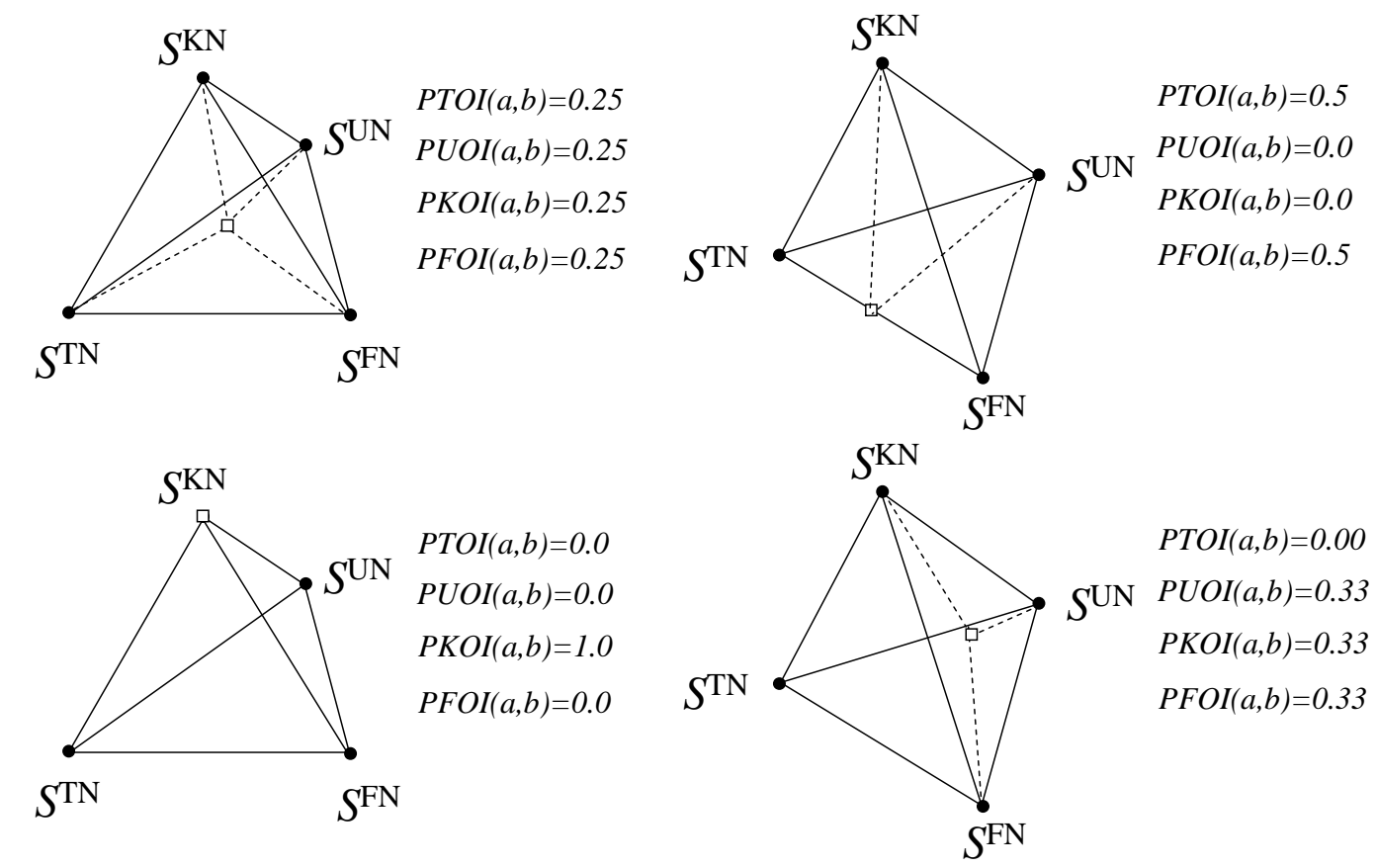

$\operatorname{PTOI}(a, b)=0.00$

$\operatorname{PUOI}(a, b)=0.33$

$\operatorname{PKOI}(a, b)=0.33$

$\operatorname{PFOI}(a, b)=0.33$

Figure 1: Representation of barycentric coordinates on a tetrahedron for four exemplary pairs of alternatives.

In the e-Appendix $\mathrm{C}$, we show how to construct a single representative set of rules by exploiting the indices $\operatorname{PTOI}(a, b), \operatorname{PUOI}(a, b), \operatorname{PKOI}(a, b)$, and $\operatorname{PFOI}(a, b)$.

\section{Ranking and Choice Exploitation Procedures}

In this section, we discuss several procedures exploiting the results obtained with all compatible MC sets of rules $\mathcal{R}^{A^{R}}$. These approaches deliver a recommendation in terms of ranking of all alternatives or in form of a subset of the potential best options. We refer to a representative sample of algorithms that can be devised, bearing in mind that a large spectrum of other exploitation procedures could be applied in this context as well. The proposed scoring procedures either directly exploit the relations $S$ and $S^{c}$ using the Net Flow Score (NFS) method (see, e.g., (Greco et al., 1998)), or transform them first into a fuzzy relation $F R$ over set $A$ (Szelag et al., 2014). The third possibility, not illustrated in this paper, consists in a separate exploitation of relations 
$S$ and $S^{c}$, leading to two separate pre-orders, which are subsequently combined into a final ranking in the spirit of ELECTRE III (Figueira et al., 2013).

\subsection{Direct Exploitation of Outranking and Non-outranking Relations}

Let us recall that given a single set of rules $\mathcal{R}$, the traditionally used NFS procedure computes for each alternative $a \in A$ a single score indicating its quality derived from the comparison with the remaining alternatives. The final ranking is determined by $\operatorname{NFS}(a, \mathcal{R})$ (the greater the score, the better), whereas the subset of the best options is formed by these with the highest scores. In the context of outcomes obtained by applying a single set of rules $\mathcal{R} \in \mathcal{R}^{A^{R}}$, for each $a \in A$ its score may be computed as (Greco et al., 2001a; Szelag et al., 2014):

$$
N F S(a, \mathcal{R})=\sum_{b \in A}|a S b|-|b S a|+\left|b S^{c} a\right|-\left|a S^{c} b\right| .
$$

Thus, the strength of $a$ is derived from outranking other alternatives and not being outranked by them, whereas its weakness comes from non-outranking other alternatives and being outranked by them. Precisely, these measures of desirability are computed so that each alternative $b \in A$ satisfying the above mentioned relations contributes with a positive or negative point to the comprehensive score of $a$. We will adapt this formula to the case of all compatible MC sets of decision rules considered together.

\section{Cumulative outranking indices}

When taking into account all compatible sets of rules $\mathcal{R}^{A^{R}}$, let us define a set of cumulative pairwise outranking and non-outranking indices:

- cumulative positive pairwise outranking (non-outranking) index expresses how much $a$ outranks all other alternatives (all other alternatives do not outrank $a$ ) for all $\mathcal{R}^{A^{R}}$ :

$$
\operatorname{CPOI}^{+}(a)=\sum_{b \in A} \operatorname{POI}(a, b) \quad\left(\operatorname{CPNOI}^{+}(a)=\sum_{b \in A} P N O I(b, a)\right)
$$

- cumulative negative pairwise outranking (non-outranking) index expresses how much all other alternatives outrank $a$ ( $a$ does not outrank all other alternatives) for all $\mathcal{R}^{A^{R}}$ :

$$
\operatorname{CPOI}^{-}(a)=\sum_{b \in A} P O I(b, a) \quad\left(C^{P N O I^{-}}(a)=\sum_{b \in A} P N O I(a, b)\right) .
$$

Thus defined positive indices support the strength of $a$, whereas the negative indices quantify its weakness. These arguments are represented graphically in Figure 2a). The above mentioned indices can be aggregated 
with a cumulative outranking index $C O I$, being the measure of the desirability of $a$ with respect to all sets of rules compatible with the DM's preferences:

$$
C O I\left(a, \mathcal{R}^{A^{R}}\right)=\operatorname{CPOI}^{+}(a)-\mathrm{CPOI}^{-}(a)+\mathrm{CPNOI}^{+}(a)-\mathrm{CPNOI}^{-}(a) .
$$

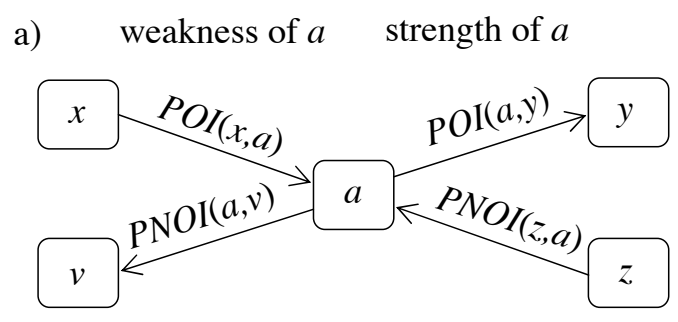

b) weakness of $a \quad$ strength of $a$

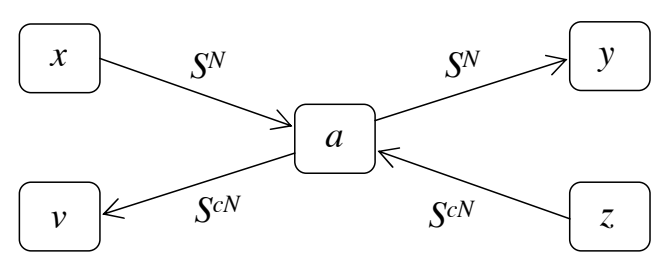

Figure 2: Arguments in favor and against the strength of alternative $a$ when referring to a) outranking indices, b) the most certain recommendation.

\section{Net Flow Score limited to the most certain recommendation}

When considering the results of applying all compatible sets of rules $\mathcal{R}^{A^{R}}$, arguments considered within the NFS procedure may be limited to the most certain recommendation only. Then, for each $a \in A$, its strength can be derived from necessary outranking over other alternatives and being necessarily non-outranked by them, whereas its weakness comes from the necessary non-outranking over other alternatives and being necessarily outranked by them. The above arguments are represented graphically in Figure $2 \mathrm{~b}$ ). Then, for each $a \in A$, NFS aggregates these arguments in the following way:

$$
N F S\left(a, \mathcal{R}^{A^{R}}\right)=\sum_{b \in A}\left|a S^{N} b\right|+\left|b S^{c N} a\right|-\left|b S^{N} a\right|-\left|a S^{c N} b\right|
$$

\section{Illustrative example (part 6): cumulative outranking indices and net flow scores limited to the most certain recommendation.}

In Table 6, we provide cumulative outranking indices (column COI; the partial results are provided in columns $\mathrm{CPOI}^{+}, \mathrm{CPNOI}^{+}, \mathrm{CPOI}^{-}$, and $\left.\mathrm{CPNOI}^{-}\right)$and net flow scores obtained when limiting the analysis to the most certain recommendation (column NFS). Rankings obtained with these aggregated measures confirm that $a_{2}, a_{3}$, and $a_{4}$ are top three alternatives, while $a_{14}, a_{15}$, and $a_{16}$ are bottom three alternatives.

\section{Rank acceptability indices}

Apart from considering the variability of the outranking and non-outranking relations illustrated in Section 4 , it is interesting to analyze the ranks that each alternative attains as a result of exploiting relations $S$ and $S^{c}$ by each compatible set of rules in $\mathcal{R}^{A^{R}}$ with NFS procedure. Precisely, the rank of $a$ (denoted by 
Table 6: Net flow scores limited to the most certain recommendation $(N F S)$ and cumulative outranking indices $(C O I)$ along with the partial results.

\begin{tabular}{|c|c|c|c|c|c|c|}
\hline & $N F S$ & COI & $\mathrm{CPOI}^{+}$ & $\mathrm{CPNOI}^{+}$ & $\mathrm{CPOI}^{-}$ & $C P N O I^{-}$ \\
\hline$a_{1}$ & $18(4)$ & $18.97(4)$ & 13.79 & 11.35 & 3.08 & 3.09 \\
\hline$a_{2}$ & $20(2)$ & $21.00(3)$ & 13.54 & 12.43 & 3.58 & 1.39 \\
\hline$a_{3}$ & $21(1)$ & $22.11(2)$ & 14.38 & 12.39 & 3.09 & 1.65 \\
\hline$a_{4}$ & $19(3)$ & $23.91(1)$ & 14.29 & 14.00 & 3.04 & 1.35 \\
\hline$a_{5}$ & $16(5)$ & $17.36(5)$ & 13.54 & 11.61 & 4.83 & 2.96 \\
\hline$a_{6}$ & $-8(10)$ & $-1.02(8)$ & 8.42 & 6.17 & 8.92 & 6.70 \\
\hline$a_{7}$ & $6(6)$ & $9.05(6)$ & 10.71 & 9.96 & 6.92 & 4.70 \\
\hline$a_{8}$ & $-8(10)$ & $-10.04(11)$ & 5.88 & 3.87 & 10.92 & 8.87 \\
\hline$a_{9}$ & $3(7)$ & $0.82(7)$ & 8.58 & 7.13 & 8.46 & 6.43 \\
\hline$a_{10}$ & $-5(8)$ & $-2.79(9)$ & 8.25 & 5.30 & 8.00 & 8.43 \\
\hline$a_{11}$ & $-7(9)$ & $-6.64(10)$ & 6.50 & 4.17 & 9.79 & 7.52 \\
\hline$a_{12}$ & $-11(13)$ & $-13.78(12)$ & 3.80 & 5.30 & 12.96 & 9.96 \\
\hline$a_{13}$ & $-10(12)$ & $-14.90(13)$ & 5.71 & 3.30 & 12.00 & 11.91 \\
\hline$a_{14}$ & $-16(14)$ & $-17.78(14)$ & 4.42 & 2.61 & 13.38 & 11.43 \\
\hline$a_{15}$ & $-17(15)$ & $-24.22(16)$ & 2.75 & 0.79 & 14.71 & 13.04 \\
\hline$a_{16}$ & $-21(16)$ & $-22.04(15)$ & 3.00 & 1.87 & 14.00 & 12.91 \\
\hline
\end{tabular}

$\operatorname{rank}(a, N F S, \mathcal{R}))$ relative to all alternatives in $A$ for a given rule set $\mathcal{R}$ is defined with the following ranking function:

$$
\begin{aligned}
& \operatorname{rank}(a, N F S, \mathcal{R})=1+\sum_{b \in A \backslash\{a\}} h(a, b, N F S, \mathcal{R}), \text { where } \\
& h(a, b, N F S, \mathcal{R})= \begin{cases}1, & \text { if } N F S(b, \mathcal{R})>N F S(a, \mathcal{R}) \\
0, & \text { otherwise. }\end{cases}
\end{aligned}
$$

Following (Kadziński and Tervonen, 2013), we define the rank acceptability index $R A I(a, k) \in[0,1]$, for alternative $a \in A$ and $\operatorname{rank} k=1, \ldots, n$, as the share of all compatible MC sets of rules that grant alternative $a$ rank $k$. Let us denote by $\mathcal{R}_{a \rightarrow k}$ a family of these sets of rules over $\mathcal{R}^{A^{R}}$, whose recommendation, when exploited with NFS, ranks alternative $a$ at $k$-th position (i.e., $\operatorname{rank}(a, N F S, \mathcal{R})=k$ ). Then, $R A I(a, k) \operatorname{can}$ be computed as the ratio of cardinalities of the families $\mathcal{R}_{a \rightarrow k}$ and $\mathcal{R}^{A^{R}}$, i.e.:

$$
R A I(a, k)=\frac{\left|\mathcal{R}_{a \rightarrow k}\right|}{\left|\mathcal{R}^{A^{R}}\right|}
$$

Let us denote the best and the worst ranks that alternative $a \in A$ can attain by $P^{*}(a)$ and $P_{*}(a)$, respectively. Rank acceptability indices can be used for computing an aggregated measure of overall desirability of $a \in A$ based on the attained ranks rather than scores. In particular, we shall consider (Lahdelma and Salminen, 2012):

- holistic acceptability index (expected rank): $H A I(a)=\sum_{k=1}^{n} k \cdot R A I(a, k)$; 
- $p$ best ranks acceptability index: $B R A I(a, p)=\sum_{k=1}^{p} k \cdot R A I(a, k)$, with $p<n$.

\section{Illustrative example (part 7): rank acceptability indices.}

Table 7 shows rank acceptability indices for all alternatives. When analyzing the extreme ranks only, $a_{1}, a_{2}$, $a_{3}, a_{4}$, and $a_{5}$ are potential top alternatives $\left(P^{*}(a)=1\right)$, with $a_{3}$ attaining the first rank for the majority of compatible sets of rules, and $a_{5}$ being the top one only for $0.3 \%$ compatible models. These two cities never fall out top 5, while $a_{1}, a_{2}$, and $a_{4}$ are ranked sixth in the worst case $\left(P_{*}(a)=6\right)$. Another six cities $\left(a_{6}-a_{11}\right)$ are ranked in the middle of the ranking for the majority of compatible sets of rules. Finally, $a_{12}-a_{16}$ are the least ranked alternatives with $a_{15}$ and $a_{16}$ being ranked at the very bottom most frequently. The ranks attained by $a_{15}, a_{16}, a_{3}, a_{5}, a_{9}$, and $a_{11}$ are the most stable (the number of different attained ranks is between 4 and 5), while the rankings of $a_{7}$ and $a_{12}$ are very unstable with, respectively, 11 and 9 different positions attained.

Note that when analyzing rank acceptability indices, we may indicate the ranks which are attained most often by the alternatives or view the distribution of $R A I s$. For example, although $a_{7}$ is ranked in the range $[2,12]$, the majority of compatible sets of rules rank it sixth $\left(R A I\left(a_{7}, 6\right)=61.2 \%\right)$. In the same spirit, a significant proportion of compatible MC sets of rules assign some other alternatives to the same rank (e.g., $R A I\left(a_{5}, 5\right)=63.7 \%, R A I\left(a_{11}, 10\right)=65.5 \%$, or $\left.R A I\left(a_{15}, 16\right)=64.5 \%\right)$. For some other cities, the range of the most frequently attained ranks is more precise than the whole ranking interval. For example, for over $92 \%$ of compatible sets of rules $a_{3}$ is ranked in top three, whereas, in general, it attains positions between 1 and 5 , while for over $80 \%$ of compatible models $a_{13}$ is ranked between 12 and 14 , while for all compatible MC sets of rules it can be eighth in the best case and the last one in the worst case.

In Table 7, we provide also holistic acceptability indices for all alternatives (column $H A I$ ). The best expected rank has been obtained for $a_{4}\left(H A I\left(a_{4}\right)=2.0\right)$, and the worst $H A I$ is attained by $a_{15}\left(H A I\left(a_{15}\right)=\right.$ 15.54). The similarities between the rankings determined by NFS, COI, and HAI, in terms of pairwise preference relations are significant. In particular, when using Kendall's $\tau$ for quantifying these similarities, the following results are obtained: $\tau\left(R_{N F S}, R_{C O I}\right)=0.89, \tau\left(R_{N F S}, R_{H A I}\right)=0.89$, and $\tau\left(R_{C O I}, R_{H A I}\right)=1.0$, where $R_{P R O C}$ is the ranking obtained with exploitation procedure $P R O C \in\{N F S, C O I, H A I\}$.

\subsection{Deriving a Fuzzy Preference Relation from Outranking and Non-outranking Relations}

Instead of directly exploiting the relations $S$ and $S^{c}$ with a scoring procedure, they can be first transformed into a single fuzzy preference relation $F R$ in the following way; for $(a, b) \in B$ and set of rules $\mathcal{R}$ :

$$
F P(\mathcal{R}, a, b)=\left[S(a, b)+\left(1-S^{c}(a, b)\right)\right] / 2 .
$$


Table 7: Rank acceptability indices (in \%) and holistic acceptability index (HAI)

\begin{tabular}{|c|c|c|c|c|c|c|c|c|c|c|c|c|c|c|c|c|c|}
\hline & 1 & 2 & 3 & 4 & 5 & 6 & 7 & 8 & 9 & 10 & 11 & 12 & 13 & 14 & 15 & 16 & $H A I$ \\
\hline$a_{1}$ & 11.8 & 12.7 & 18.1 & 25.5 & 16.3 & 15.6 & 0.0 & 0.0 & 0.0 & 0.0 & 0.0 & 0.0 & 0.0 & 0.0 & 0.0 & 0.0 & 3.69 \\
\hline$a_{3}$ & 21.6 & 29.7 & 40.8 & 5.4 & 2.5 & 0.0 & 0.0 & 0.0 & 0.0 & 0.0 & 0.0 & 0.0 & 0.0 & 0.0 & 0.0 & 0.0 & 2.38 \\
\hline$a_{5}$ & 0.3 & 4.2 & 8.2 & 23.6 & 63.7 & 0.0 & 0.0 & 0.0 & 0.0 & 0.0 & 0.0 & 0.0 & 0.0 & 0.0 & 0.0 & 0.0 & 4.47 \\
\hline$a_{6}$ & 0.0 & 0.0 & 0.0 & 0.0 & 0.0 & 6.0 & 40.1 & 21.7 & 22.3 & 5.6 & 2.9 & 0.7 & 0.0 & 0.0 & 0.0 & 0.0 & 7.92 \\
\hline$a_{7}$ & 0.0 & 2.0 & 1.6 & 2.4 & 11.2 & 61.2 & 9.1 & 6.7 & 2.4 & 1.3 & 0.3 & 1.8 & 0.0 & 0.0 & 0.0 & 0.0 & 6.18 \\
\hline$a_{10}$ & 0.0 & 0.0 & 0.0 & 0.0 & 0.0 & 5.8 & 11.6 & 25.9 & 35.9 & 9.2 & 9.4 & 2.2 & 0.0 & 0.0 & 0.0 & 0.0 & 8.68 \\
\hline$a_{11}$ & 0.0 & 0.0 & 0.0 & 0.0 & 0.0 & 0.0 & 0.0 & 0.4 & 9.2 & 65.4 & 21.0 & 4.0 & 0.0 & 0.0 & 0.0 & 0.0 & 10.19 \\
\hline$a_{12}$ & 0.0 & 0.0 & 0.0 & 0.0 & 0.0 & 0.0 & 0.0 & 2.2 & 10.5 & 1.4 & 17.8 & 19.2 & 25.9 & 11.2 & 8.0 & 3.8 & 12.27 \\
\hline$a_{13}$ & 0.0 & 0.0 & 0.0 & 0.0 & 0.0 & 0.0 & 0.0 & 0.3 & 2.0 & 3.8 & 6.9 & 33.7 & 19.7 & 27.9 & 4.7 & 0.9 & 12.70 \\
\hline$a_{14}$ & 0.0 & 0.0 & 0.0 & 0.0 & 0.0 & 0.0 & 0.0 & 0.0 & 0.0 & 0.0 & 3.4 & 12.3 & 33.7 & 33.9 & 16.1 & 0.5 & 13.49 \\
\hline
\end{tabular}

Note that the above defined transformation implies that $F P(\mathcal{R}, a, b)=1 / 2$ whenever $a S^{\mathcal{U}} b$ and $a S^{\mathcal{K}} b$. Although $S^{\mathcal{U}}$ and $S^{\mathcal{K}}$ represent different concepts, they can both be perceived as an intermediate level between $S^{\mathcal{F}}$ and $S^{\mathcal{T}}$. Nevertheless, while a transformation (21) is already well-established in the literature (see, e.g., (Szelag et al., 2014)), FP may be any fuzzy relation over B. For its further processing with some ranking method, it is important that $F P$ has no structural properties. It holds for our case, since $F P(\mathcal{R}, a, b)$ depends only on the considered rule set $\mathcal{R}$, which, in turn, does not generally depend on $A$ (Szelag et al., 2014).

Although there exist many ranking methods that can be used for exploiting a fuzzy relation over $B$ (for a review, see (Szelag et al., 2014)), one of the most frequently used is "sum in favor", defined in the following way:

$$
S F(a, \mathcal{R})=\sum_{b \in A} F P(\mathcal{R}, a, b)=\sum_{b \in A} 1 \cdot\left|a S^{\mathcal{T}} b\right|+\frac{1}{2} \cdot\left(\left|a S^{\mathcal{U}} b\right|+\left|a S^{\mathcal{K}} b\right|\right)+0 \cdot\left|a S^{\mathcal{F}} b\right|
$$

This method represents a group of Net Flow Rules which are characterized by the most desirable properties, thus, being considered as the best ranking procedure for exploitation of FP (Szelag et al., 2014). When taking into account all compatible sets of rules $\mathcal{R}^{A^{R}}$, let us define a comprehensive sum in favor as follows:

$$
C S F\left(a, \mathcal{R}^{A^{R}}\right)=1 \cdot \operatorname{PTOI}(a, b)+\frac{1}{2} \cdot(\operatorname{PUOI}(a, b)+\operatorname{PKOI}(a, b))+0 \cdot \operatorname{PFOI}(a, b)
$$

Note that above reference to different outranking indices allows to express CSF in a concise form. However, they are not directly applied within a scoring procedure, which instead exploits a fuzzy relation $F P(\mathcal{R}, a, b)$ for each $\mathcal{R} \subseteq \mathcal{R}^{A^{R}}$. Moreover, since for each of these rule sets application of $\operatorname{SF}(a, \mathcal{R})$ results in a weak order over $A$, we may analyze rank acceptability indices analogously as in Section 5.1. 


\section{Dealing with Incompatibility of the Preference Information}

When using value- or outranking-based MCDA methods, in case of inconsistency of the DM's preference information, two alternative approaches are employed. If the DM accepts that some of her/his statements will not be reproduced by any preference model instance, the analysis is performed while controlling the value of an acceptable total error. Otherwise, the troublesome pieces of preference information responsible for the incompatibility need to be identified with Mixed-Integer Linear Programming (MILP), and then removed or revised.

The framework of DRSA offers different possibilities for dealing with inconsistency. When referring to the certain decision rules generated from the lower approximations, we take into account a consistent part of the DM's preference information. However, we may also consider possible decision rules derived from the upper approximations. In this way, we investigate consequences of the provided preference information while accounting for the inconsistency. Obviously, in case of inconsistency the analyst should decide whether to consider either certain or possible rules, or both of them. In any case, since the sets of rules do differ, the results of their application on the set of alternatives differ as well.

\section{Illustrative example (part 8): sets of certain and possible rules.}

For the purpose of illustration, let us assume that the DM provided a different ranking for reference alternatives:

$$
\text { Cracow }\left(a_{6}\right) \succ \text { Poznan }\left(a_{2}\right) \succ \text { Ostroleka }\left(a_{16}\right) \succ \operatorname{Lomza}\left(a_{13}\right) \text {. }
$$

The underlying pairwise comparisons are inconsistent with respect to the dominance relation, which means that some pairs for which the DM required $S$ are dominated by pairs for which (s)he claimed $S^{c}$. The lower and upper approximations of these relations are as follows:

$$
\begin{array}{r}
\underline{P}(S)=\left\{\left(a_{6}, a_{6}\right),\left(a_{2}, a_{2}\right),\left(a_{16}, a_{16}\right),\left(a_{13}, a_{13}\right),\left(a_{6}, a_{16}\right),\left(a_{6}, a_{13}\right),\left(a_{2}, a_{16}\right),\left(a_{2}, a_{13}\right)\right\} \\
\bar{P}(S)=\left\{\left(a_{6}, a_{6}\right),\left(a_{2}, a_{2}\right),\left(a_{16}, a_{16}\right),\left(a_{13}, a_{13}\right),\left(a_{6}, a_{16}\right),\left(a_{6}, a_{13}\right),\left(a_{2}, a_{16}\right),\left(a_{2}, a_{13}\right),\right. \\
\left.\left(a_{6}, a_{2}\right),\left(a_{2}, a_{6}\right),\left(a_{16}, a_{13}\right),\left(a_{13}, a_{16}\right)\right\}, \\
\underline{P}\left(S^{c}\right)=\left\{\left(a_{16}, a_{6}\right),\left(a_{16}, a_{2}\right),\left(a_{13}, a_{6}\right),\left(a_{13}, a_{2}\right)\right\}, \\
\bar{P}\left(S^{c}\right)=\left\{\left(a_{16}, a_{6}\right),\left(a_{16}, a_{2}\right),\left(a_{13}, a_{6}\right),\left(a_{13}, a_{2}\right),\left(a_{6}, a_{2}\right),\left(a_{2}, a_{6}\right),\left(a_{16}, a_{13}\right),\left(a_{13}, a_{16}\right)\right\} .
\end{array}
$$

In Table 8, we present selected compatible certain and possible rules obtained with Algorithm 3 provided in the e-Appendix B. These are all rules which are contained in the minimal sets of rules given in Table 9. For depicting the exemplary recommendation that can be obtained with all MC sets of certain and possible 
rules, we present selected aggregated measures in Table 10. These are net flow scores, cumulative outranking indices, expected ranks, and extreme ranks. Their comparison reveals slight differences in values of these measures and/or the respective ranks. In particular, when comparing the pairs of rankings obtained with NFS, COI, and HAI, with certain or possible rules, the similarities quantified with Kendall's $\tau$ are equal to, respectively, 0.89, 0.94, and 1.0 .

Table 8: Selected compatible certain and possible minimal rules.

All rules used in the MC sets of certain rules

"at most" decision rules

$r_{S^{c}}^{1, C}$

$r_{S^{c}}^{2, C}$

$r_{S^{c}}^{3, C}$

$r_{S^{c}}^{4, C}$

$r_{S^{c}}^{1, P}$

$r_{S^{c}}^{2, P}$

$r_{S^{c}}^{3, P}$

$r_{S^{c}}^{4, P}$ if $\Delta_{5}(a, b) \leq-182.0$ then $a S^{c} b$

if $\Delta_{4}(a, b) \leq-0.84$ then $a S^{c} b$

if $\Delta_{3}(a, b) \leq 43.0$ and $\Delta_{5}(a, b) \leq-2.0$ then $a S^{c} b$

if $\Delta_{1}(a, b) \leq-208.0$ and $\Delta_{4}(a, b) \leq-0.41$ then $a S^{c} b$ "at least" decision rules

$r_{S}^{1, C} \quad$ if $\Delta_{5}(a, b) \geq 182.0$ then $a S b$

$r_{S}^{2, C} \quad$ if $\Delta_{4}(a, b) \geq 0.84$ then $a S b$

$r_{S}^{3, C} \quad$ if $\Delta_{3}(a, b) \geq-43.0$ and $\Delta_{4}(a, b) \geq 0.0$ then $a S b$

$r_{S}^{4, C} \quad$ if $\Delta_{3}(a, b) \geq-43.0$ and $\Delta_{5}(a, b) \geq 0.0$ then $a S b$

All rules used in the MC sets of possible rules

"at most" decision rules

if $\Delta_{5}(a, b) \leq-2.0$ then $a S^{c} b$

if $\Delta_{4}(a, b) \leq-0.41$ then $a S^{c} b$

if $\Delta_{3}(a, b) \leq-14.0$ and $\Delta_{5}(a, b) \leq 119.0$ then $a S^{c} b$

if $\Delta_{3}(a, b) \leq-14.0$ and $\Delta_{3}(a, b) \leq 0.64$ then $a S^{c} b$ "at least" decision rules

$r_{S}^{1, P} \quad$ if $\Delta_{5}(a, b) \geq-119.0$ then $a S b$

$r_{S}^{2, P} \quad$ if $\Delta_{4}(a, b) \geq 0.0$ then $a S b$

$r_{S}^{3, P} \quad$ if $\Delta_{1}(a, b) \geq-106.0$ and $\Delta_{4}(a, b) \geq-0.64$ then $a S b$

$r_{S}^{4, P} \quad$ if $\Delta_{3}(a, b) \geq 0.0$ and $\Delta_{4}(a, b) \geq-0.64$ then $a S b$

Table 9: All MC sets of rules for the lower and upper approximations of relations $S$ and $S^{c}$. $\mathcal{R} \frac{P}{C, m r c}\left(S^{c}\right)=3 \mathrm{MC}$ sets of rules for $\underline{P}\left(S^{c}\right)=\left\{r_{S^{c}}^{1, C}\right\},\left\{r_{S^{c}}^{2, C}, r_{S^{c}}^{3, C}\right\},\left\{r_{S^{c}}^{2, C}, r_{S^{c}}^{4, C}\right\}$

$\mathcal{R}_{C}^{P}, m r c=4 \mathrm{MC}$ sets of rules for $\underline{P}(S)=\left\{r_{S}^{1, C}, r_{S}^{3, C}\right\},\left\{r_{S}^{1, C}, r_{S}^{4, C}\right\},\left\{r_{S}^{2, C}, r_{S}^{3, C}\right\},\left\{r_{S}^{2, C}, r_{S}^{4, C}\right\}$

$\mathcal{R}_{P, m r c}^{\bar{P}\left(S^{c}\right)}=4 \mathrm{MC}$ sets of rules for $\bar{P}\left(S^{c}\right)=\left\{r_{S^{c}}^{1, P}, r_{S^{c}}^{3, P}\right\},\left\{r_{S^{c}}^{1, P}, r_{S^{c}}^{4, P}\right\},\left\{r_{S^{c}}^{2, P}, r_{S^{c}}^{3, P}\right\},,\left\{r_{S^{c}}^{2, P}, r_{S^{c}}^{4, P}\right\}$

$\mathcal{R}_{P, m r c}^{\bar{P}(S)}=3 \mathrm{MC}$ sets of rules for $\bar{P}(S)=\left\{r_{S}^{1, P}\right\},\left\{r_{S}^{2, P}, r_{S}^{3, P}\right\},\left\{r_{S}^{2, P}, r_{S}^{4, P}\right\}$

Table 10: Comparison of aggregated measures obtained with all compatible MC sets of certain and possible rules.

\begin{tabular}{|c|c|c|c|c|c|c|c|c|}
\hline & \multicolumn{2}{|c|}{$N F S$ (rank) } & \multicolumn{2}{|c|}{$C O I$ (rank) } & \multicolumn{2}{|c|}{$H A I$} & \multicolumn{2}{|c|}{$P^{*}(a)-P_{*}(a)$} \\
\hline & certain & possible & certain & possible & certain & possible & certain & possible \\
\hline$a_{1}$ & $13(5)$ & $11(6)$ & $13.58(4)$ & $11.92(4)$ & 5.33 & 4.75 & $4-7$ & $3-7$ \\
\hline$a_{2}$ & $10(6)$ & $13(5)$ & $9.28(6)$ & $11.50(6)$ & 5.25 & 5.08 & $3-7$ & $3-7$ \\
\hline$a_{3}$ & $22(2)$ & $21(3)$ & $19.17(2)$ & $19.50(2)$ & 3.00 & 2.83 & $2-5$ & $2-6$ \\
\hline$a_{4}$ & $25(1)$ & $26(1)$ & $24.17(1)$ & $24.83(1)$ & 1.58 & 1.33 & $1-5$ & $1-3$ \\
\hline$a_{5}$ & $16(4)$ & $15(4)$ & $13.17(5)$ & $11.92(4)$ & 4.50 & 5.08 & $1-9$ & $1-9$ \\
\hline$a_{6}$ & $-2(8)$ & $-2(9)$ & $-1.86(8)$ & $-1.42(9)$ & 7.66 & 8.41 & $6-9$ & $7-10$ \\
\hline$a_{7}$ & $21(3)$ & $22(2)$ & $17.25(3)$ & $16.92(3)$ & 2.75 & 3.67 & $1-5$ & $1-7$ \\
\hline$a_{8}$ & $-10(12)$ & $-11(11)$ & $-10.69(13)$ & $-11.25(13)$ & 12.33 & 11.91 & $11-14$ & $11-13$ \\
\hline$a_{9}$ & $3(7)$ & $5(7)$ & $0.69(7)$ & $1.58(7)$ & 8.75 & 7.50 & $6-11$ & $4-13$ \\
\hline$a_{10}$ & $-6(10)$ & $-11(11)$ & $-6.89(10)$ & $-9.67(11)$ & 11.08 & 11.08 & $8-14$ & $8-14$ \\
\hline$a_{11}$ & $-11(13)$ & $-12(13)$ & $-9.43(12)$ & $-9.42(10)$ & 10.83 & 11.17 & $10-12$ & $10-13$ \\
\hline$a_{12}$ & $-5(9)$ & $1(8)$ & $-4.97(9)$ & $-0.42(8)$ & 8.83 & 8.75 & $4-15$ & $4-14$ \\
\hline$a_{13}$ & $-21(14)$ & $-21(14)$ & $-16.83(14)$ & $-16.50(14)$ & 13.58 & 13.58 & $12-15$ & $11-15$ \\
\hline$a_{14}$ & $-8(11)$ & $-9(10)$ & $-7.50(11)$ & $-10.17(12)$ & 10.58 & 11.08 & $3-15$ & $5-16$ \\
\hline$a_{15}$ & $-22(15)$ & $-23(15)$ & $-17.36(15)$ & $-19.25(15)$ & 14.83 & 14.58 & $11-16$ & $10-16$ \\
\hline$a_{16}$ & $-25(16)$ & $-25(16)$ & $-21.75(16)$ & $-20.08(16)$ & 15.08 & 15.16 & $13-16$ & $13-16$ \\
\hline
\end{tabular}

The approach presented in this paper is also valid in case of Variable Consistency Dominance-based Rough Set Approach (VC-DRSA) (Błaszczyński et al., 2009; Szelag et al., 2014). VC-DRSA produces greater lower 
approximations than those computed by traditional DRSA, containing alternatives or pairs of alternatives strongly, though not necessarily, related with the approximated sets. To quantify the degree of this relation, the following consistency measure $\epsilon_{T}: B \rightarrow[0,1]$ for a pair $(a, b)$ with respect to $T=S$ or $S^{c}$ is used:

$$
\epsilon_{S}(a, b)=\left|S^{c} \cap D_{2}^{+}(a, b)\right| /\left|S^{c}\right| \quad \text { and } \quad \epsilon_{S^{c}}(a, b)=\left|S \cap D_{2}^{-}(a, b)\right| /|S|
$$

Thus defined, the value of $\epsilon_{T}(a, b)$ which is close to one indicates low consistency of pair $(a, b)$ with relation $T$. The revised definitions of lower approximations of $S$ and $S^{c}$ incorporating the above defined consistency measures are as follows (see (Szelag et al., 2014)):

$$
\underline{P}(S)=\left\{(a, b) \in S: \epsilon_{S}(a, b) \leq \theta_{S}\right\} \text { and } \underline{P}\left(S^{c}\right)=\left\{(a, b) \in S^{c}: \epsilon_{S^{c}}(a, b) \leq \theta_{S^{c}}\right\}
$$

with consistency thresholds $\theta_{S}, \theta_{S^{c}} \in[0,1)$. For discussion on different consistency measures, see (Błaszczyński et al., 2009). When inducing compatible decision rules, we need to control their consistency measure $\varepsilon_{T} \in[0,1]$ defined as follows:

$$
\varepsilon_{S}\left(r_{S}\right)=\left|S ^ { c } \cap \left\|\Phi\left(r_{S}\right)|\| /| S^{c} \mid \quad \text { and } \quad \varepsilon_{S^{C}}\left(r_{S^{C}}\right)=\left|S \cap \| \Phi\left(r_{S^{C}}\right)\right||| /|S|\right.\right.
$$

where $\Phi\left(r_{T}\right)$ is a condition part of rule $r_{T}$. The rules subsequently induced from the lower approximations of relation $T$ need to satisfy the same consistency level as pairs of alternatives contained in $\underline{P}(T)$, i.e., $\varepsilon_{T}\left(r_{T}\right) \leq \theta_{T}$. Thus, a suitable extension of Algorithm 3 provided in the e-Appendix B to the VC-DRSA setting is straightforward.

\section{Toward Ensuring Consistency with Preference Information in the Final Ranking}

Although pairwise comparisons provided by the DM are reproduced by the induced sets of rules, it is wellknown that when exploited with NFS the order for some pairs of reference alternatives may be inversed (for a more thorough discussion, see (Szelag et al., 2014)). For example, even though the DM required $a S b\left(a S^{c} b\right)$, $N F S(a)<N F S(b)(N F S(a)>N F S(b))$. Dealing with this problem, we may follow the approach proposed by Kadziński et al. (2012) for outranking-based PROMETHEE method by distinguishing the construction and exploitation levels of the outranking relation. The former refers to the comparison of performances of $a$ and $b$, and is modeled with decision rules. The latter refers to the ranks attained by $a$ and $b$, and is modeled with respect to their net flow scores. To avoid the problem of rank reversals at the exploitation level, we may take into account only these sets of rules that reproduce DM's preferences at both levels instead of construction 
level only. Referring to the exploitation level, we may also account for different types of holistic preference information such as rank-related requirements (Kadziński et al., 2013).

\section{Decision Aiding with the Proposed Approach}

Ranking and choice decisions can be aided with the proposed approach through the iterative process illustrated in Figure 3. The process begins by defining the problem in Step 1. This requires specification of a set of alternatives $A$, a set of criteria $G$, and the performance matrix. Then, in Step 2 pairwise comparisons for a small subset of reference alternatives are elicited (see Section 2).

Step 3 consists of structuring the provided preference information using the lower and upper approximation of relations $S$ and $S^{c}$ (see Section 2). In case of inconsistency (i.e., when $B n_{G}(S)=B n_{G}\left(S^{c}\right) \neq \emptyset$ ), the DM may revise her preference information or decide to continue the analysis with inconsistency (see Section 6). Then, in Step 4, we construct a set of all MC sets of rules by first generating an exhaustive set of all minimal decision rules, and then finding all minimal covers of pairs of alternatives with ILP (see Section 3).

Step 5 consists of building a ranking/choice recommendation. First, we construct the necessary and possible relations and compute the respective probabilistic indices (see Section 4). Depending on the selected logic (either two or four valued), these results may refer to, respectively, outranking and non-outranking or true, false, unknown, and contradictory outranking. We may also apply different scoring procedures which either directly exploit the outranking relations or first transform them into a single fuzzy relation (see Section 5). The results that can be derived from such analysis express the probabilities that alternatives attain particular ranks or provide a complete order with respect to, e.g., cumulative outranking indices, holistic acceptability indices, net flow scores, or comprehensive sum in favor scores. These results may be additionally enriched with a selection of a representative set of rules (see e-Appendix C). Obviously, in Step 5 we may use only a small subset of results that may be delivered within the proposed framework.

The above procedures should be used only in case a numerical score has to be assigned to each alternative (Roy and Słowiński, 2013). Obviously, the analyst needs to be aware that they may deliver different rankings. Since there is no single best approach that can be adopted in the context of all real-world problems, the selection of a particular procedure should be motivated by its properties and underlying logic. In particular, $C O I$ is appropriate for aggregation of the numerical scores derived from the exploitation of outranking relation determined individually by each compatible set of rules. Further, NFS is relevant for an analogous aggregation limited, however, only to the most certain recommendation provided by all sets of rules considered jointly. Finally, $H A I$ should be used when the analyst cares more about the ordinal ranks (rather than the numerical scores) stemming from the application of each rule set. Additionally, each of these procedures may 


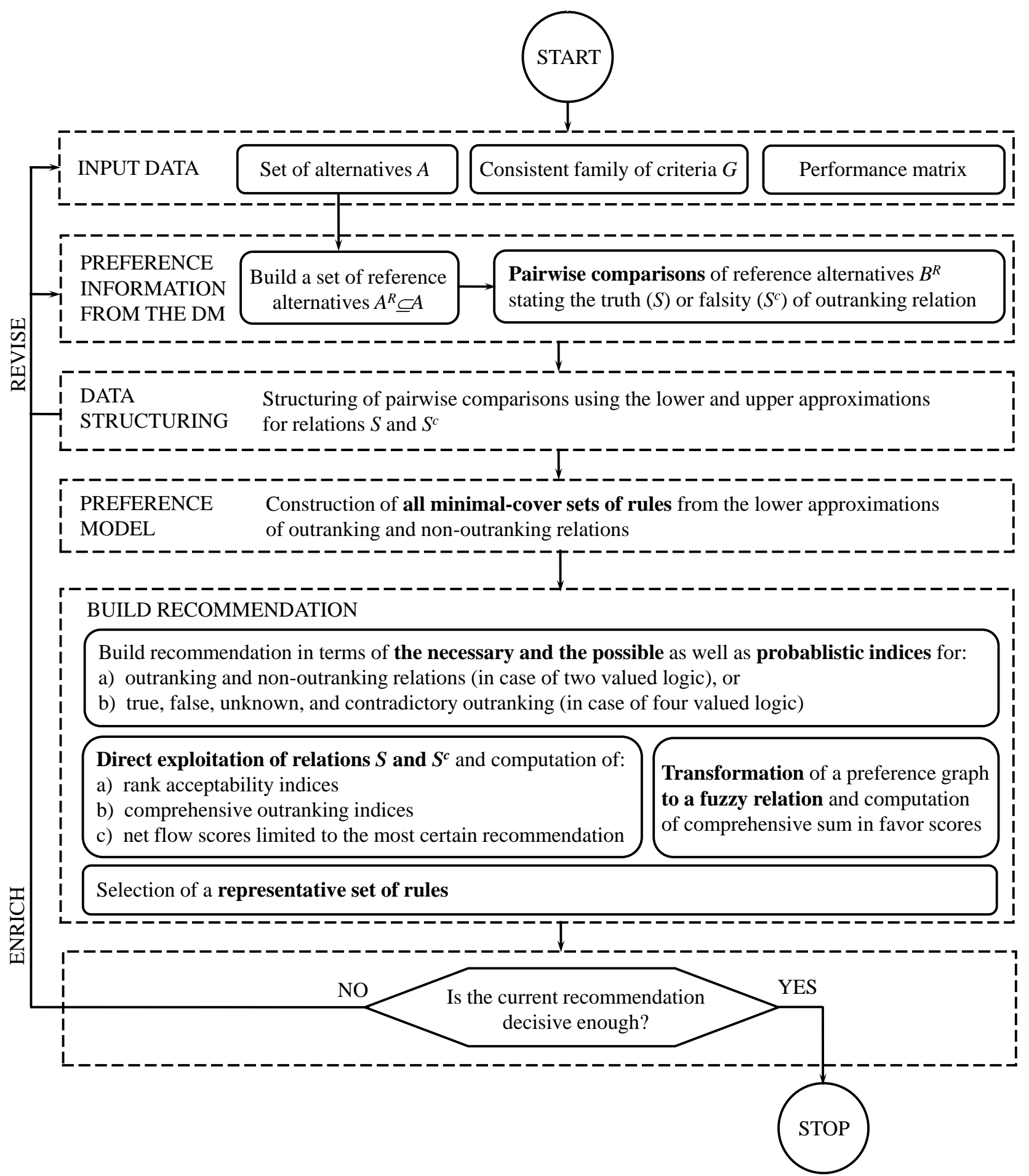

STEP 1

STEP 2

STEP 3

STEP 4

Figure 3: Decision aiding process for the proposed approach. 
be useful for making the respective outcome of robustness analysis more descriptive and comprehensible (i.e., $N F S$ for $S^{N}$ and $S^{c N}, C O I$ for $P O I \mathrm{~s}$ and $P N O I \mathrm{~s}$, or $H A I$ for $\left.R A I \mathrm{~s}\right)$. It simply brings closer the alternatives which on average are similar in terms of a given perspective.

All provided results are verified in Step 6; if the DM is satisfied with the recommendation, the process ends. Otherwise, one should revise the input data and/or enrich the preference information. The suggested procedure is to provide new pairwise comparisons for these pairs for which we observe the possible though non-necessary relation at the current stage of interaction.

Let us emphasize that our proposal implements a constructivist approach postulated in MCDA (Corrente et al., 2013; Roy, 2010). We do not assume that a preference model is an entity that is pre-existing in the DM's mind which need to be discovered by the algorithm. Indeed, neither the objective truth/recommendation nor the "true" form/values of the preference model exist. On the contrary, we assume that the model is constructed in course of an interaction with the method. We intentionally force the DM to confront her/his value system with a variety of results of applying the inferred preference model on the set of alternatives rather than provide a clear-cut ranking/choice. In this way, (s)he should gain insights on her/his value system and better understand the employed method.

This contrasts with the discovery of statistically validated preference patterns which is postulated in machine learning (see, e.g., (Fürnkranz and Hüllermeier, 2010)). As a result, the problems of referring directly to the estimation paradigm within an empirical comparison or measuring the method's effectiveness in terms of a misranking error, are ill-founded in our context. This issue is widely discussed by Corrente et al. (2013).

\section{Conclusions}

We presented a new approach for dealing with multiple criteria ranking problems. The approach derives from Dominance-based Rough Set Approach, and considers all MC sets of minimal rules compatible with the pairwise comparisons provided by the DM. Then, the method provides acceptability indices for pairwise preference relations as well as for all pairs of alternatives and ranks. These correspond to the estimates of the probabilities that one alternative outranks (does not outrank) the other or attains some particular rank. Such probabilistic results may be used to obtain the necessary and possible outranking relations and extreme ranks. These relations are confirmed by all or at least one compatible MC set of rules, whereas the extreme ranks for each alternative are obtained with Net Flow Score exploitation of the most and least advantageous sets of rules. All these results may be aggregated to obtain a comprehensive evaluation of the alternatives in terms of a recommendation provided by all compatible sets of rules. In particular, we proposed to consider a cumulative outranking index (average net flow score) or a holistic acceptability index (expected rank). We 
also discussed several extensions of the main approach. These include selection of the representative MC set of rules as well as an application of possible rules along with the certain ones. The introduced method was illustrated using an example of ranking Polish cities according to their innovativeness.

The complexity of generating all compatible rules is exponential and identification of the minimal sets of rules by solving a set cover problem is NP-hard. Thus, the proposed approach requires a considerable amount of computational time. For example, the execution time for our illustrative problem on Intel Atom CPU D325 $1.80 \mathrm{GHz}$ with $4 \mathrm{~GB}$ RAM was about 60 seconds. This enables dealing with modestly sized ranking and choice problems considered in MCDA where it is unrealistic to assume to the DM provides more than few tens of holistic judgments (Spliet and Tervonen, 2014). However, the high computational complexity prevents using the proposed method in the context of big data problems where the preference information comes from the observation of users' behavior rather than from direct questioning of the DM.

Nevertheless, the benefits of using all compatible sets of rules far outweigh the complexity of the proposed algorithms. Even solely with the illustrative study, we prove how much information is neglected by other existing approaches and how arbitrary is the recommendation provided by them. In any case, to address the existence of multiple sets of rules and avoid heavy calculations, we may refer to some sequential covering algorithm (see, e.g., (Błaszczyński et al., 2009), (Greco et al., 2001b), or (Szelag et al., 2014)) and run it a certain number of times. Each run should be either perturbed, e.g., by not choosing always the best candidate for the elementary condition, or limited to a subset of criteria (e.g., the reducts (Shao and Leung, 2014; Susmaga, 2014)). This would allow induction of multiple compatible sets of rules, which may be further analyzed in terms of robustness of provided recommendation in the usual way.

The whole approach can be extended in several ways, in particular, by:

- considering only satisfactory sets of rules being consistent with some pre-defined user requirements;

- adapting to group decision problems (Chakhar and Saad, 2012; Greco et al., 2006);

- accounting for the decision under uncertainty (Greco et al., 2010; Słowiński et al., 2014) formulated as a multiple criteria ranking problem;

- identification of minimal sets of the provided pairwise comparisons that imply the obtained possible and necessary preference relations (Kadziński et al., 2014a),

- implementing a Map-Reduce version of the algorithms for generating all compatible rules and using some heuristic procedures for generating the minimal covers. 


\section{Acknowledgments}

The first author acknowledges financial support from the Polish National Science Center (grant SONATA, no. DEC-2013/11/D/ST6/03056).

\section{References}

An, L., Tong, L., 2009. Learning rules from pairwise comparison table. In: Deng, H., Wang, L., Wang, F., Lei, J. (Eds.), Artificial Intelligence and Computational Intelligence. Vol. 5855 of Lecture Notes in Computer Science. Springer, pp. 18-27.

An, L., Tong, L., 2010. Rough approximations based on intersection of indiscernibility, similarity and outranking relations. Knowledge-Based Systems 23 (6), 555 - 562.

Augeri, M., Cozzo, P., Greco, S., 2015. Dominance-based rough set approach: An application case study for setting speed limits for vehicles in speed controlled zones. Knowledge-Based Systems (in press), http://dx.doi.org/10.1016/j.knosys.2015.07.010.

Błaszczyński, J., Greco, S., Słowiński, R., Szelag, M., 2009. Monotonic variable consistency rough set approaches. International Journal of Approximate Reasoning 50 (7), 979-999.

Błaszczyński, J., Słowiński, R., Szelag, M., 2011. Sequential covering rule induction algorithm for variable consistency rough set approaches. Information Sciences 181 (5), 987-1002.

Bouyssou, D., Perny, P., 1992. Ranking methods for valued preference relations: A characterization of a method based on leaving and entering flows. European Journal of Operational Research 61 (12), 186 - 194.

Brans, J., Mareschal, B., 1994. The PROMCALC \& GAIA decision support system for multicriteria decision aid. Decision Support Systems 12, 297 - 310.

Chakhar, S., Saad, I., 2012. Dominance-based rough set approach for groups in multicriteria classification problems. Decision Support Systems 54 (1), 372-380.

Corrente, S., Greco, S., Kadziński, M., Słowiński, R., 2013. Robust ordinal regression in preference learning and ranking. Machine Learning 93 (2-3), 381-422.

Corrente, S., Greco, S., Słowiński, R., 2012. Multiple criteria hierarchy process in robust ordinal regression. Decision Support Systems 53 (3), 660-674. 
Dias, L., Clímaco, J., 2002. Aiding Decisions with Multiple Criteria - Essays in Honor of Bernard Roy. In: Bouyssou, D., Jacquet-Lagrèze, E., Perny, P., Słowiński, R., Vanderpooten, D., Vincke, P. (Eds.), New Advances in Multiple Criteria Decision Analysis. Kluwer, pp. 175-193.

Dias, L., Lamboray, C., 2010. Extensions of the prudence principle to exploit a valued outranking relation. European Journal of Operational Research 201 (3), 828 - 837.

Figueira, J. R., Greco, S., Roy, B., Słowiński, R., 2013. An overview of ELECTRE methods and their recent extensions. Journal of Multi-Criteria Decision Analysis 20 (1-2), 61-85.

Fortemps, P., Greco, S., Słowiński, R., 2008. Multicriteria decision support using rules that represent roughgraded preference relations. European Journal of Operational Research 188 (1), 206 - 223.

Fürnkranz, J., Hüllermeier, E., 2010. Preference learning and ranking by pairwise comparison. In: Fürnkranz, J., Hüllermeier, E. (Eds.), Preference Learning. Springer, pp. 65-82.

Greco, S., Kadziński, M., Mousseau, V., Słowiński, R., 2011. ELECTRE ${ }^{\text {GKMS }}$ Robust ordinal regression for outranking methods. European Journal of Operational Research 214 (1), 118-135.

Greco, S., Matarazzo, B., Słowiński, R., 1999a. Rough approximation of a preference relation by dominance relations. European Journal of Operational Research 117, 63 - 83.

Greco, S., Matarazzo, B., Słowiński, R., 1999b. The use of rough sets and fuzzy sets in mcdm. In: Gal, T., Stewart, T., Hanne, T. (Eds.), Multicriteria Decision Making. Vol. 21 of International Series in Operations Research \& Management Science. Springer US, pp. 397-455.

Greco, S., Matarazzo, B., Słowiński, R., 2001a. Rough sets theory for multicriteria decision analysis. European Journal of Operational Research 129, $1-47$.

Greco, S., Matarazzo, B., Słowiński, R., 2005. Decision rule approach. In: Multiple Criteria Decision Analysis: State of the Art Surveys. Vol. 78 of International Series in Operations Research \& Management Science. Springer New York, pp. 507-555.

Greco, S., Matarazzo, B., Słowiński, R., 2006. Dominance-based rough set approach to decision involving multiple decision makers. In: Greco, S., Hata, Y., Hirano, S., Inuiguchi, M., Miyamoto, S., Nguyen, H., Słowiński, R. (Eds.), Rough Sets and Current Trends in Computing. Vol. 4259 of Lecture Notes in Computer Science. Springer Berlin Heidelberg, pp. 306-317. 
Greco, S., Matarazzo, B., Słowiński, R., 2010. Dominance-based rough set approach to decision under uncertainty and time preference. Annals of Operations Research 176 (1), 41-75.

Greco, S., Matarazzo, B., Słowiński, R., Stefanowski, J., 2001b. An algorithm for induction of decision rules consistent with the dominance principle. In: Ziarko, W., Yao, Y. Y. (Eds.), Rough Sets and Current Trends in Computing, , Second International Conference, RSCTC 2000 Banff, Canada, October 16-19, 2000,. Vol. 2005 of Lecture Notes in Computer Science. Springer, pp. 304-313.

Greco, S., Matarazzo, B., Słowiński, R., Tsoukias, A., 1998. Exploitation of a rough approximation of the outranking relation in multicriteria choice and ranking. In: Stewart, T., van den Honert, R. (Eds.), Trends in Multicriteria Decision Making. Vol. 1998 of Lecture Notes in Economics and Mathematical Systems. Springer, Berlin, pp. 45-60.

Greco, S., Mousseau, V., Słowiński, R., 2008. Ordinal regression revisited: multiple criteria ranking using a set of additive value functions. European Journal of Operational Research 191 (2), 415-435.

Greco, S., Słowiński, R., Zielniewicz, P., 2013. Putting Dominance-based Rough Set Approach and Robust Ordinal Regression together. Decision Support Systems 54 (2), 891-903.

Hazen, G., 1986. Partial information, dominance, and potential optimality in multiattribute utility theory. Operations Research 34 (2), $296-310$.

Huang, B., Li, H.-X., Wei, D.-K., 2012. Dominance-based rough set model in intuitionistic fuzzy information systems. Knowledge-Based Systems 28, 115 - 123.

Jacquet-Lagreze, E., Siskos, J., 1982. Assessing a set of additive utility functions for multicriteria decisionmaking, the $\{\mathrm{UTA}\}$ method. European Journal of Operational Research 10 (2), 151 - 164.

Kadziński, M., Corrente, S., Greco, S., Słowiński, R., 2014a. Preferential reducts and constructs in robust multiple criteria ranking and sorting. OR Spectrum 36 (4), 1021-1053.

Kadziński, M., Greco, S., Słowiński, R., 2012. Extreme ranking analysis in robust ordinal regression. Omega $40(4), 488-501$.

Kadziński, M., Greco, S., Słowiński, R., 2013. RUTA: a framework for assessing and selecting additive value functions on the basis of rank related requirements. Omega 41 (4), 735-751.

Kadziński, M., Greco, S., Słowiński, R., 2014b. Robust ordinal regression for dominance-based rough set approach to multiple criteria sorting. Information Sciences 283, 211-228. 
Kadziński, M., Tervonen, T., 2013. Robust multi-criteria ranking with additive value models and holistic pair-wise preference statements. European Journal of Operational Research 228 (1), 169-180.

Keeney, R., Raiffa, H., 1976. Decisions with Multiple Objectives: Preferences and Value Trade-offs. Cambridge University Press, Cambridge.

Lahdelma, R., Salminen, P., 2012. The shape of the utility or value function in stochastic multicriteria acceptability analysis. OR Spectrum 34 (4), $785-802$.

Lee, K., Park, K., Kim, S., 2002. Dominance, potential optimality, imprecise information, and hierarchical structure in multi-criteria analysis. Computers \& Operations Research 29 (9), 1267-1281.

Li, S., Li, T., Hu, J., 2015a. Update of approximations in composite information systems. Knowledge-Based Systems 83, 138-148.

Li, S., Li, T., Liu, D., 2013. Incremental updating approximations in dominance-based rough sets approach under the variation of the attribute set. Knowledge-Based Systems 40, $17-26$.

Li, S., Li, T., Zhang, Z., Chen, H., Zhang, J., 2015b. Parallel computing of approximations in dominance-based rough sets approach. Knowledge-Based Systems (in press), -, http://dx.doi.org/10.1016/j.knosys.2015.05.003.

Pawlak, Z., Słowiński, R., 1994. Rough set approach to multi-attribute decision analysis. European Journal of Operational Research $72(3), 443-459$.

Roy, B., 1990. The outranking approach and the foundations of ELECTRE methods. In: Bana e Costa, C. (Ed.), Readings in Multiple Criteria Decision Aid. Springer-Verlag, Berlin, pp. 155-183.

Roy, B., 2010. Two conceptions of decision aiding. International Journal of Multicriteria Decision Making (IJMCDM) 1 (1), 74-79.

Roy, B., Słowiński, R., 2013. Questions guiding the choice of a multicriteria decision aiding method. EURO Journal on Decision Processes 1 (1-2), 69-97.

Rzeczpospolita, 2012. Ranking of municipalities (in Polish). Rzeczpospolita (rp.pl/rankingsamorzadow2012), Warsaw.

Shao, M.-W., Leung, Y., 2014. Relations between granular reduct and dominance reduct in formal contexts. Knowledge-Based Systems 63, 1-11. 
Słowiński, R., Greco, S., Matarazzo, B., 2012. Rough set and rule-based multicriteria decision aiding. Pesquisa Operacional $32(2), 213-269$.

Słowiński, R., Kadziński, M., Greco, S., 2014. Robust ordinal regression for dominance-based rough set approach under uncertainty. In: Kryszkiewicz, M., Cornelis, C., Ciucci, D., Medina-Moreno, J., Motoda, H., Raś, Z. (Eds.), Rough Sets and Intelligent Systems Paradigms. Vol. 8537 of Lecture Notes in Computer Science. Springer, pp. $77-87$.

Spliet, R., Tervonen, T., 2014. Preference inference with general additive value models and holistic pair-wise statements. European Journal of Operational Research 232 (3), 607-612.

Susmaga, R., 2014. Reducts and constructs in classic and Dominance-based Rough Sets Approach. Information Sciences 271, 45-64.

Szelag, M., Greco, S., Słowiński, R., 2013. Aiding Decisions with Multiple Criteria - Essays in Honor of Bernard Roy. In: Doumpos, M., Grigoroudis, E. (Eds.), Multicriteria decision aid and artificial intelligence: links, theory and applications. Wiley-Blackwell, London, pp. 127-160.

Szelag, M., Greco, S., Słowiński, R., 2014. Variable consistency Dominance-based Rough Set Approach to preference learning in multicriteria ranking. Information Sciences 277, 525-552.

Tsoukias, A., Vincke, P., 1995. A new axiomatic foundation of partial comparability. Theory and Decision 39 (1), 79-114.

Vazirani, V., 2001. Approximation algorithms. Springer, New York, USA.

White, C., Sage, A., Dozono, S., 1984. A model of multiattribute decisionmaking and trade-off weight determination under uncertainty. IEEE Transactions on Systems, Man and Cybernetics 14 (2), 223 - 229. 